\title{
Targeting neuronal gap junctions in mouse retina offers neuroprotection in glaucoma
}

\author{
Abram Akopian, Sandeep Kumar, Hariharasubramanian Ramakrishnan, Kaushambi Roy, Suresh Viswanathan, \\ and Stewart A. Bloomfield \\ Department of Biological and Vision Science, State University of New York College of Optometry, New York, New York, USA.
}

\begin{abstract}
The progressive death of retinal ganglion cells and resulting visual deficits are hallmarks of glaucoma, but the underlying mechanisms remain unclear. In many neurodegenerative diseases, cell death induced by primary insult is followed by a wave of secondary loss. Gap junctions (GJs), intercellular channels composed of subunit connexins, can play a major role in secondary cell death by forming conduits through which toxic molecules from dying cells pass to and injure coupled neighbors. Here we have shown that pharmacological blockade of GJs or genetic ablation of connexin 36 (Cx36) subunits, which are highly expressed by retinal neurons, markedly reduced loss of neurons and optic nerve axons in a mouse model of glaucoma. Further, functional parameters that are negatively affected in glaucoma, including the electroretinogram, visual evoked potential, visual spatial acuity, and contrast sensitivity, were maintained at control levels when Cx36 was ablated. Neuronal GJs may thus represent potential therapeutic targets to prevent the progressive neurodegeneration and visual impairment associated with glaucoma.
\end{abstract}

\section{Introduction}

Glaucoma is a neurodegenerative disease often associated with elevated intraocular pressure (IOP) and characterized by the progressive loss of retinal neurons and optic nerve axons, leading to vision impairment $(1,2)$. Glaucoma likely has multivariate etiology, and so the exact mechanisms triggering its development and progression remain elusive (3). The use of IOP-lowering drugs, the current mainstay treatment, is often insufficient to prevent progressive visual loss in glaucoma patients. Recent studies of potential treatments have therefore shifted to assessment of neuroprotective strategies to promote neuronal survivability and thereby preserve function (3-6).

In many neurodegenerative diseases in the CNS, including glaucoma, there is an initial loss of vulnerable neurons related directly to the primary insult, followed by a progressive, secondary cell loss $(7,8)$. There is now strong evidence that intercellular communication through gap junctions (GJs) plays a major role in secondary or so-called bystander cell death (9-11). In this scheme, toxic molecules are transferred intercellularly from dying cells to neighbors through communicating GJs, thereby producing a wave of ancillary cell degeneration $(12,13)$. As the CNS locus with arguably the highest expression of GJs, bystander-mediated neuronal cell death in retina has been implicated in a number of pathologies, including excitotoxicity, retinitis pigmentosa, and ischemic retinopathy $(12,14-17)$.

Damage to the optic nerve due to elevated IOP and the loss of retinal ganglion cells (RGCs) are key sequelae of glaucoma, but amacrine cells (ACs), interneurons critical to retinal signaling, also

Authorship note: A. Akopian and S. Kumar are co-first authors. Conflict of interest: The authors have declared that no conflict of interest exists. Submitted: November 23, 2016; Accepted: April 27, 2017. Reference information: / Clin Invest. 2017;127(7):2647-2661. https://doi.org/10.1172/JCI91948. appear to show significant injury (18-22). In mice, nearly all the RGC subtypes are homologously coupled via GJs (23), thereby providing the structural substrate for progressive, bystander-mediated cell death. Most RGCs also couple heterologously to ACs (23), suggesting that primary RGC loss in glaucomatous eyes can lead to AC loss through GJ-mediated secondary cell death (12). Indeed, a recent study showed that the most vulnerable ACs in experimental glaucoma are those that are coupled to RGCs (22). Together, these data suggest that GJ-mediated secondary cell death may ultimately be responsible for the majority of neuronal loss and the resultant impaired visual function associated with glaucoma.

Here we demonstrate that pharmacological blockade of neuronal GJs or genetic ablation of connexin 36 (Cx36), the most abundantly expressed GJ subunit in retinal neurons, provides significant protection of the retina and optic nerve against damage in a mouse model of glaucoma. Further, this structural protection results in preservation of visual function as indicated by electrophysiological measures of electroretinogram (ERG) and visual evoked potential (VEP) and behavioral assessment of spatial acuity and contrast sensitivity. Our results thus indicate that neuronal GJs play a principal role in progressive cell loss and visual impairment associated with glaucoma, thereby identifying them as potential targets for novel neuroprotective therapies.

\section{Results}

IOP elevation in a mouse model of glaucoma in control and experimental animals. To induce experimental glaucoma in mice, we adopted and modified a microbead occlusion model to elevate IOP (24). In control C57BL/6 mice, intracameral injection of microbeads accumulated in the iridocorneal angle and Schlemm's canal (Figure 1A), resulting in a significant elevation of IOP within 1 week (Figure 1B). The IOP declined slowly over the next 3 weeks, and so a 
A

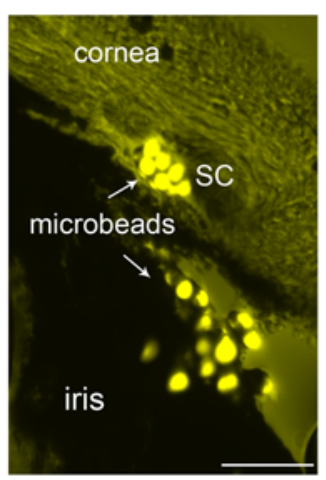

D

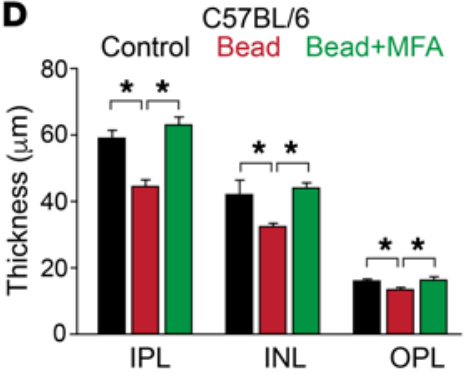

B

Control Bead Bead+MFA

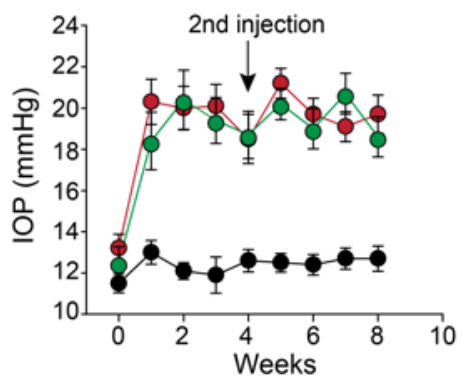

$\mathbf{E}$

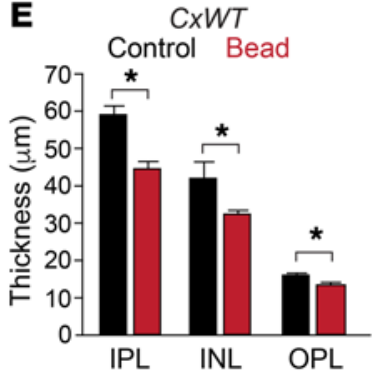

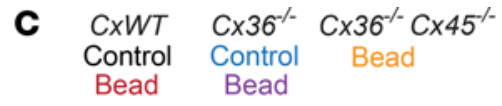
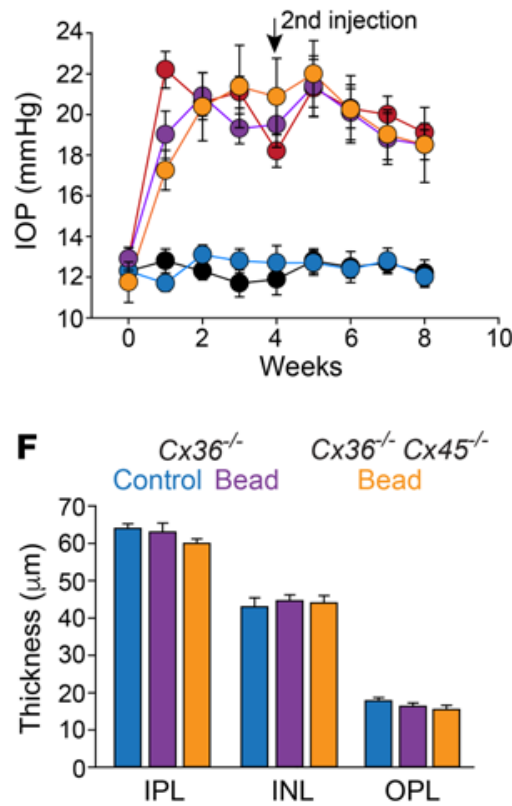

Figure 1. Blockade of GJs suppresses gross retinal injury in glaucomatous eyes. (A) Photomicrograph shows accumulation of microbeads in the iridocorneal angle and Schlemm's canal (SC) 8 weeks after intracameral injection. Scale bar: $100 \mu \mathrm{m}$. (B) C57BL/6 mouse data showing sustained IOP elevation 8 weeks after initial microbead injection followed by a second injection at week 4 (arrow) in comparison with control eyes (sham injection of PBS). MFA application did not affect the elevated IOP observed in microbead-injected eyes ( $n=10$ eyes per group). (C) Elevation of IOP in different mouse strains following microbead injections ( $n=10$ eyes per group). Sham injections produced no elevation of IOP. (D) Thickness of individual retinal layers in glaucomatous retinas with or without MFA treatment ( $n=6$ retinas per group). MFA application prevents thinning of layers in glaucomatous eyes. (E) Thickness of retinal layers in CxWT mice with and without microbead injections ( $n=6$ retinas per group). (F) Preservation of retinal layer thickness in glaucomatous retinas of $C \times 36^{-1-}$ or C $\times 36^{-1-} C \times 45^{-1-}$ mice $\left(n=6\right.$ retinas per group). Data are presented as mean \pm SEM. ${ }^{*} P<0.05$, Student's $t$ test for B, C, and $\mathbf{E}$ and 1 -way ANOVA followed by Tukey's multiple comparisons test for $\mathbf{D}$ and $\mathbf{F}$.

second injection of microbeads was performed at 4 weeks, which maintained the elevated IOP for at least 8 weeks (Figure 1B). We next determined whether the microbead occlusion method was effective in elevating IOP in our experimental mice in which GJs were blocked pharmacologically or connexin subunits were ablated genetically. Blockade of GJs was carried out by application of meclofenamic acid (MFA), a nonsteroidal antiinflammatory (25), which is a potent, while connexin-nonspecific, GJ antagonist (26). In initial control experiments, we determined whether MFA application via an osmotic minipump was injurious to the retina. At a concentration that effectively uncoupled neuronal GJs in the retina, we found that MFA did not alter the gross or cellular structure of the retina based on measures of cell counts in the ganglion cell layer (GCL) and thicknesses of the nuclear and plexiform layers in WT C57BL/6 mice (Supplemental Figure 1; supplemental material available online with this article; https://doi.org/10.1172/JCI91948DS1).

Application of microbeads to eyes of C57BL/6 mice in which GJs were blocked with MFA produced an elevation in IOP that was indistinguishable from those measured in untreated animals at each time point over the 8-week postinjection period (Figure 1B). Similarly, microbead injections into the eyes of $\mathrm{C} \times 36^{--}$or $\mathrm{C} \times 36^{--} \mathrm{Cx} 45^{-/-}$double knockout mice, in which the most abundantly expressed neuronal connexin subunits are ablated (13), produced an elevated IOP that mirrored in amplitude and time course that measured in glaucomatous C57BL/6 mice or wild-type littermates (CxWT) (Figure 1, B and C). Sham intracameral injections of PBS did not markedly alter the average IOP from that seen in control animals (Figure 1, B and C). Overall, these data indicated that the microbead occlusion method was effective in elevating IOP to induce experimental glaucoma in both control and experimental animals.

Gross retinal injury in experimental glaucoma is significantly reduced by GJ blockade or ablation. We next assessed gross structural changes of glaucomatous retinas 8 weeks after initial microbead injection. Morphometric analysis of DAPI-labeled vertical retinal sections revealed a statistically significant decrease in the thickness of the inner plexiform layer (IPL), inner nuclear layer (INL), and outer plexiform layer (OPL) of glaucomatous C57BL/6 and CxWT mice compared with control values (Figure 1, D and E). All measurements of the thickness of individual retinal layers were performed at a midperipheral eccentricity, 1.5-2.0 mm from the optic nerve head. In contrast, no significant reduction in the thickness of individual retinal layers was detected in glaucomatous retinas of MFA-treated C57BL/6 mice or $C \times 36^{-/}$and $C x 36^{--} \mathrm{C} x 45^{-/-}$ mice relative to values observed in control retinas (Figure 1, D and F). The similar results found for the $\mathrm{C} \times 36^{--}$and $\mathrm{C} \times 36^{--} \mathrm{C} \times 45^{--}$ mice indicated that deletion of $\mathrm{Cx} 45$-expressing GJs provided no additional protection over deletion of those expressing Cx36.

In glaucoma, as in other neurodegenerative disorders, glial cells show abnormal activity, termed reactive gliosis, which results in an upregulation of glial fibrillary acidic protein (GFAP) expres- 
A

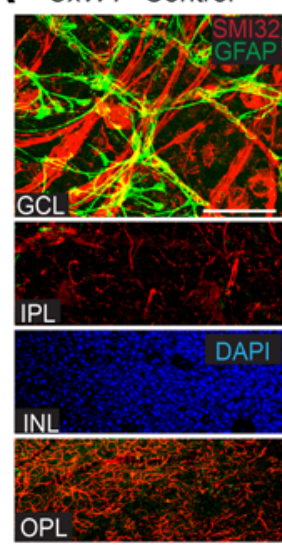

CxWT Bead

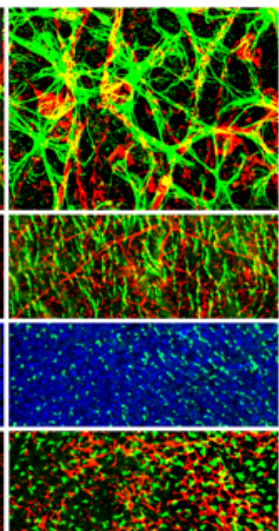

CxWT Bead + MFA

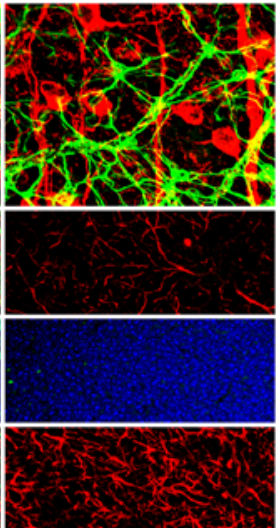

$\mathrm{C} \times 36^{-1-}$
B
CxWT

Control Bead Bead+MFA
Control Bead
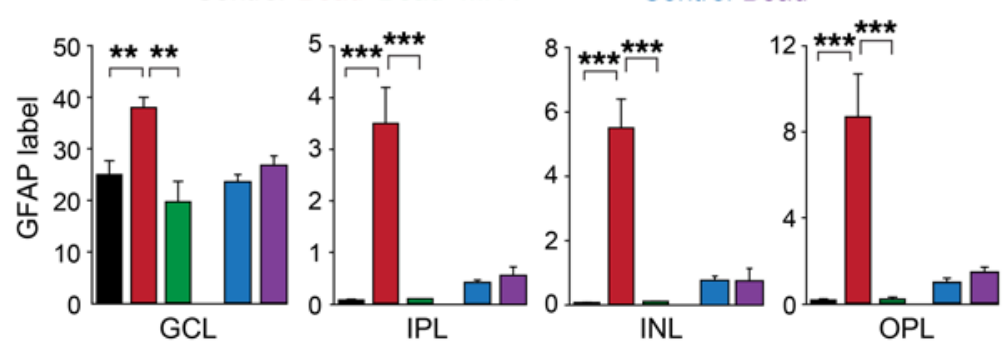

CX36 $6^{-/-}$Control

C $\times 36^{-/-}$Bead
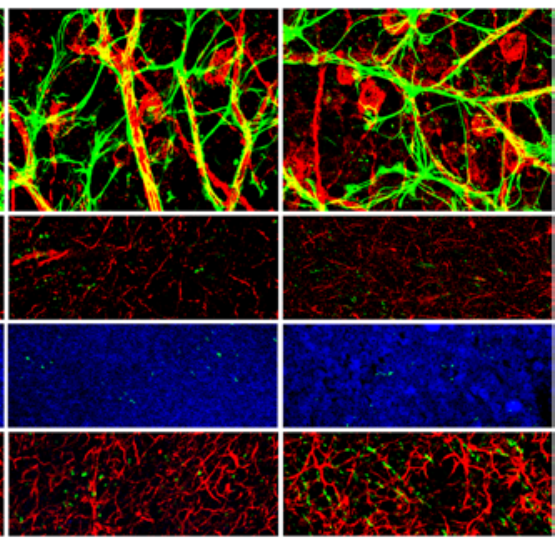

C CxWT Cx36/- Cx36/- $\mathrm{C} \times 45^{-/-}$ Control Bead Bead Bead

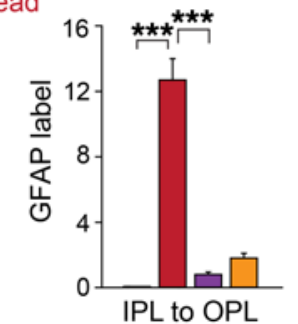

Figure 2. Reactive gliosis in retinas of microbead-injected mice is significantly reduced by GJ blockade/ablation. (A) Confocal images of retinal layers stained for GFAP, SMI32, and DAPI in control and glaucomatous retinas. Scale bar: $50 \mu \mathrm{m}$ in all panels. Z-stack: 7 sections, 3- $\mu \mathrm{m}$ steps. (B) GFAP expression in the retinal layers of $C \times W T$ and $C \times 36^{-/-}$mouse retinas under different conditions ( $n=6$ retinas per group). (C) GFAP labeling in retinal sections from control and microbead-injected $C x W T$ ( $n=5$ retinas), $C \times 36^{-1-}\left(n=5\right.$ retinas), and $C \times 36^{-1-} C \times 45^{-1-}$ mice $(n=3$ retinas). GFAP expression is presented as percentage of immunolabeling per area. Data are presented as mean $\pm \mathrm{SEM} .{ }^{* *} P<0.01,{ }^{* * *} P<0.001,1$-way ANOVA followed by Tukey's multiple comparisons test.

sion in astrocytes and Müller cells in retina $(27,28)$. Under control conditions, GFAP immunofluorescence was confined exclusively to astrocytes in the GCL, with no detectable immunolabeling in the IPL, INL, or OPL (Figure 2A). Eight weeks after microbead injection, astrocytes showed a significant increase in GFAP labeling as evidenced by the appearance of a thick network of processes in the GCL (Figure 2, A and B). A more dramatic increase in GFAP immunolabeling was observed in the IPL, INL, and OPL of glaucomatous retinas due to an upregulation of GFAP in Müller cell processes (Figure 2, A and B). In contrast, the reactive gliosis in glaucomatous retinas of MFA-treated mice was markedly suppressed relative to that observed in untreated microbead-injected retinas (Figure 2, A and B). Similarly, retinas from microbead-injected $C \times 36^{-/-}$mice exhibited a significantly lower degree of reactive gliosis throughout compared with that observed in their CxWT littermates (Figure 2, A and B). Blockade of retinal GJs or ablation of Cx36 clearly reduced the overall structural damage to the retina brought on by elevated IOP. However, genetic ablation of both Cx36 and Cx45 showed no further improvement in the suppression of the reactive gliosis in vertical sections of glaucomatous retinas when compared with that seen in $\mathrm{Cx}_{36} 6^{-/}$mice (Figure 2C).

Blockade or ablation of GJs protects GCL neurons in experimental glaucoma. To evaluate the role of GJs in the progressive loss of retinal neurons in experimental glaucoma, we performed a comparative histochemical analysis of glaucomatous retinas in the presence and absence of gap junctional communication. Retinal whole mounts under control and experimental conditions were immunostained for SMI32, which has been shown to label somata, dendrites, and axons of a subset of large RGCs, identified as $\alpha$ cells (refs. 29, 30, and Figure 3, A and E). In glaucomatous retinas of C57BL/6 mice we found a marked decrease in the density of SMI32-positive axons in comparison with control eyes (Figure 3, A and B). In contrast, blockade of GJs with MFA in microbead-injected eyes maintained the density of SMI32-positive axons at a level comparable to that seen in control retinas (Figure 3, A and B). To evaluate RGC loss in microbead-injected retinas in the presence and absence of GJ communication, we immunostained retinal whole mounts with the selective RGC marker BRN3A (31), under control conditions and 8 weeks after microbead injection with or without MFA treatment (Figure 3C). Cell counts were performed in 4 selected fields of identical size per retina at a midperipheral eccentricity of 1.5-2.0 mm from the optic disk (Figure 3C, inset). Quantification revealed significantly higher RGC survival in retinas of microbead-injected eyes from MFA-treated mice compared with those untreated (Figure 3D).

We next examined the impact of glaucomatous insult on $\alpha$-RGCs by immunostaining retinal whole mounts for SMI32 under control and experimental conditions (Figure 3E). Eight weeks after microbead injection we observed a significant reduction in the number of SMI32-positive RGC somata and dendrites (Figure 3, E and F). Blockade of GJs with MFA again provided significant protection to microbead-injected retinas as indicated by 
A

C

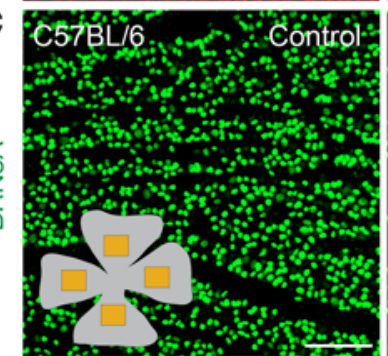

E

C57BL/6

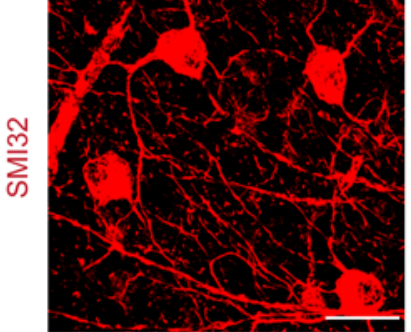

G

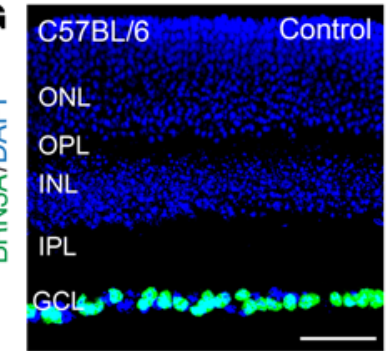

H $C \times W T$
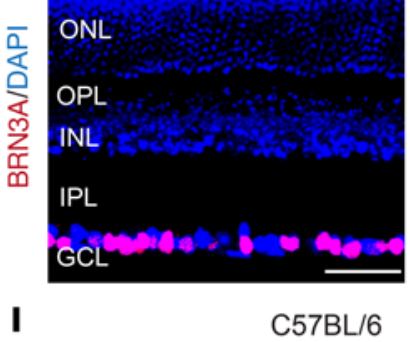

*** Control Bead
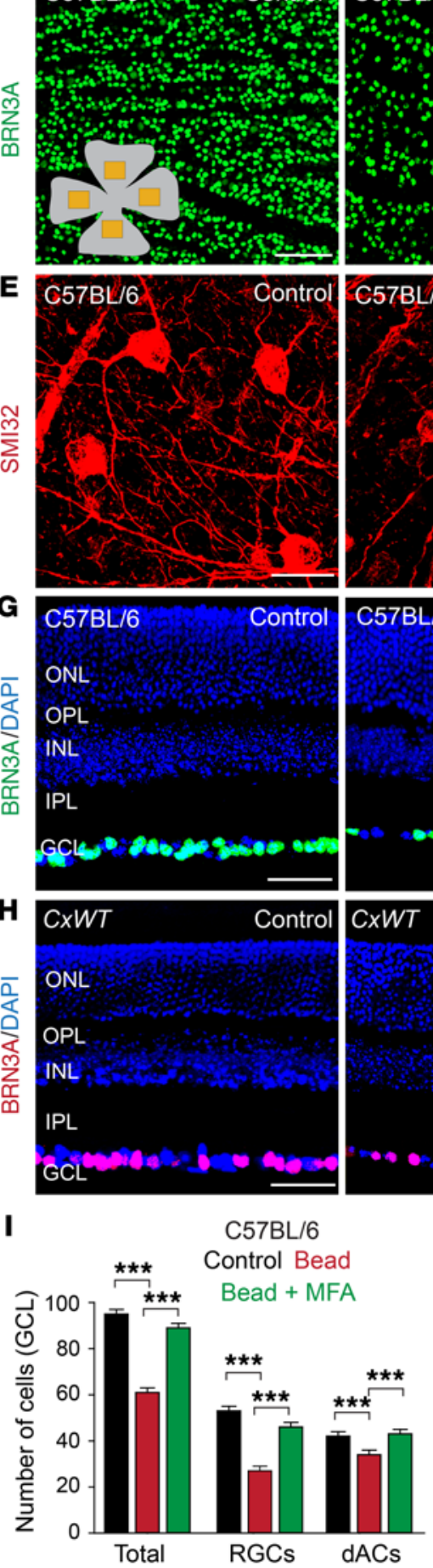
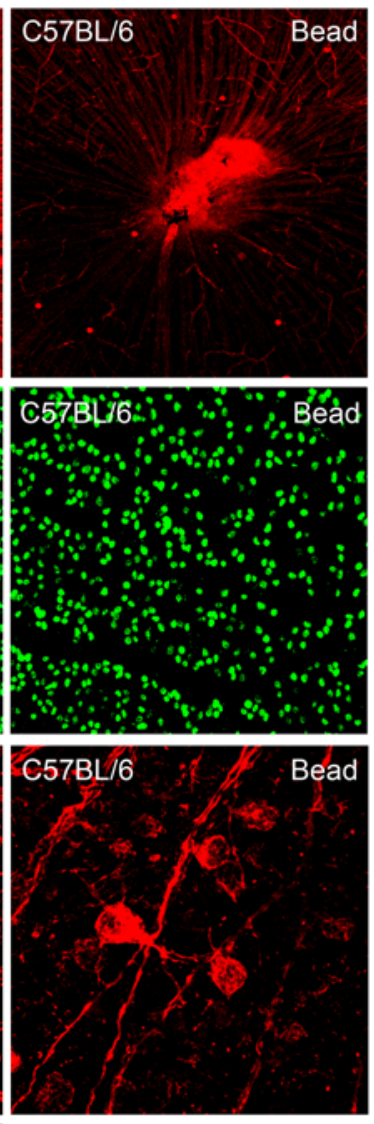

C57BL/6

Bead
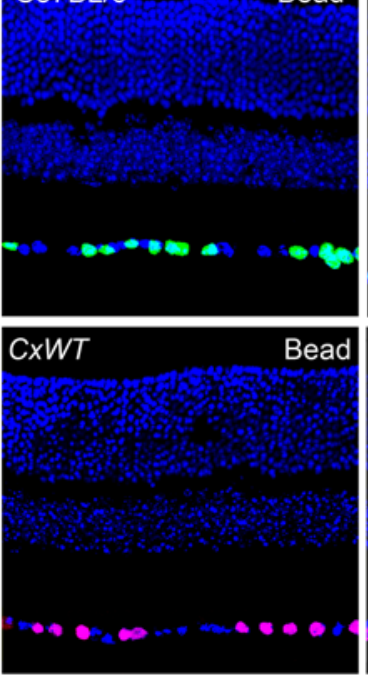

J

Control Bead

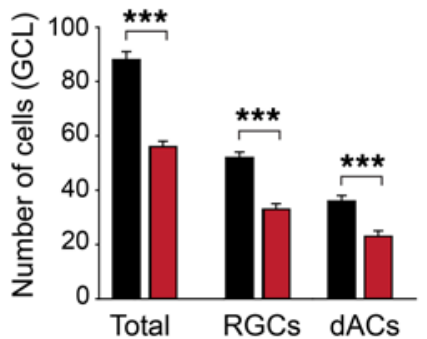

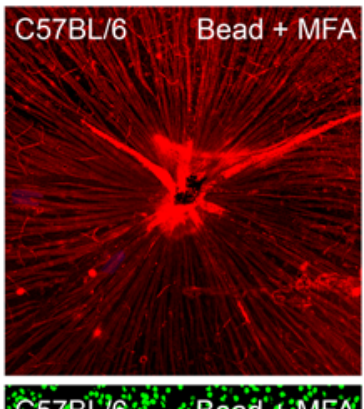

C57Bi $76 \%$ Bead
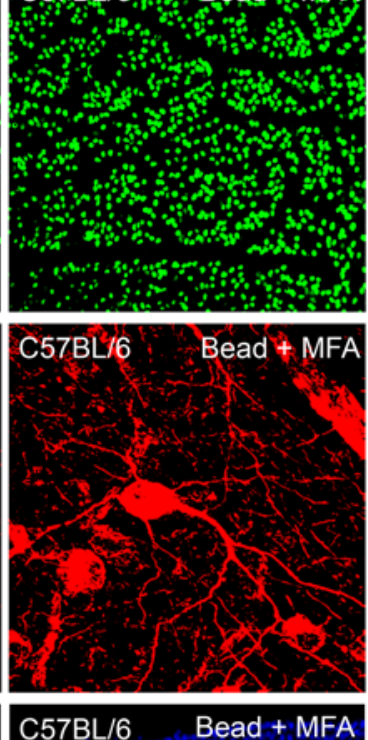

Bead + MFA
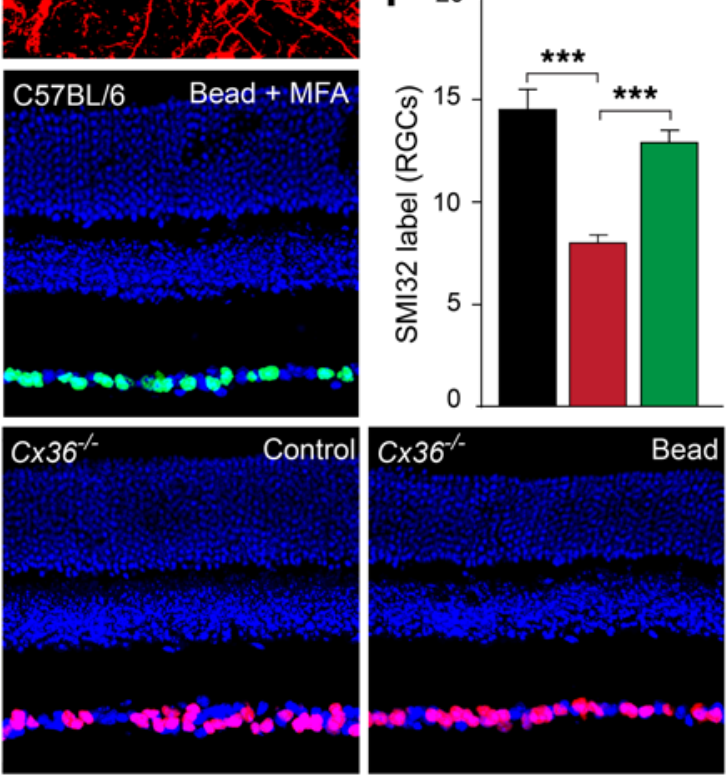

$\mathbf{K}$

C $\times 36^{-1-}$

Control Bead Bead

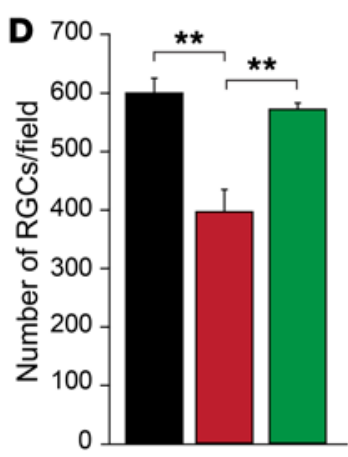

F 20

Bead

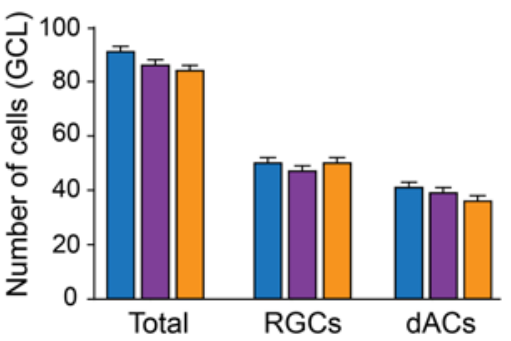


Figure 3. Blockade or ablation of GJs protects RGCs and their axons in experimental glaucoma. (A) Immunofluorescence images of SMI32-pos-

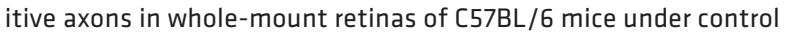
conditions and 8 weeks after microbead injections with or without MFA treatment ( $n=6$ retinas per group). Scale bar: $200 \mu \mathrm{m}$ for all panels. (B) SMI32-labeled axons as percentage of coverage per $300 \times 300 \mu \mathrm{m}$ retinal area from each quadrant, averaged across retinas. (C) Confocal images of whole-mount retinas with BRN3A-labeled RGCs under conditions represented in $\mathbf{A}$. All images and measures were taken from the midperiphery of the retina, $1.5-2.0 \mathrm{~mm}$ from the optic disk (inset). Scale bar: $100 \mu \mathrm{m}$ for all panels. (D) Quantification of RGCs performed for $600 \times 600 \mu \mathrm{m}$ area in 4 quadrants from the midperipheral retina and averaged for retinas per each control and experimental condition ( $n=3$ retinas per group). (E) Images of SMI32-positive $\alpha$-RGC somata and dendrites under conditions described in $\mathbf{A}$ ( $n=6$ retinas per group). Scale bar: 25 $\mu \mathrm{m}$ for all panels. Z-stack: 15 sections, 2- $\mu \mathrm{m}$ steps. (F) Quantification of SMI32-positive $\alpha$-RGC somata under conditions represented in $\mathbf{E}$. (C) C57BL/6 retinal sections processed for BRN3A immunoreactivity and counterstained with DAPI in control and MFA-treated or untreated glaucomatous eyes. Scale bar: $50 \mu \mathrm{m}$ for all panels. Z-stack: 5 sections 2 - $\mu \mathrm{m}$ steps. (H) Images of retinal sections from control and microbead-injected eyes of $C x W T$ and $C x 36^{-/-}$mice. Scale bar: $50 \mu \mathrm{m}$ for all panels. (I) Number of DAPI-stained (total) cells, BRN3A-positive RGCs, and $\mathrm{dACs}$ in the GCL of retinas from $\mathrm{C57BL} / 6$ mice under conditions described in $\mathbf{E}$ ( $n=14$ retinas for all counts). (J) Number of total cells, RGCs, and dACs in the GCL of CxWT in control eyes and 8 weeks after microbead injection ( $n=10$ retinas per group). Z-stack: 5 sections, $2-\mu \mathrm{m}$ steps. (K) Number of total cells, RGCs, and dACs in the GCL of $C \times 36^{-1-}$ ( $n=12$ retinas) and $C \times 36^{-/-} C x 45^{-/-}(n=5$ retinas) mice in controls and 8 weeks after microbead injection. All data are presented as mean \pm SEM. ${ }^{* *} P<0.001,{ }^{* *} P>0.01$, Student's $t$ test for J and 1-way ANOVA followed by Tukey's multiple comparisons test for $\mathbf{B}, \mathbf{D}, \mathbf{F}, \mathbf{I}$, and $\mathbf{K}$.

maintenance of both the number of SMI32-positive RGCs and the soma/dendritic architecture that were comparable to those in control eyes (Figure 3, $\mathrm{E}$ and $\mathrm{F}$ ).

Approximately one-half of the neurons in the GCL of the mouse retina are displaced amacrine cells (dACs) (32). To distinguish between RGCs and dACs in terms of their loss in glaucoma, we immunostained vertical retinal sections taken from midperipheral retina with BRN3A to identify RGCs and then counterstained with the nuclear dye DAPI to assess the total number of cells in the GCL (Figure 3G). The number of dACs was computed as the difference between DAPI-positive nuclei and BRN3A-positive cells in the GCL as we described in a recent study (22). We observed a significant decrease in both BRN3A-positive RGCs and dACs in C57BL/6 mice measured at 8 weeks after the initial microbead injection (Figure 3I). However, the loss of DAPI-positive cells was greater than that of BRN3A-positive RGCs, indicating a loss of dACs in the GCL. In contrast, blockade of GJs with MFA provided significant protection of both cell types in microbead-injected retinas (Figure 3, G and H). Induction of glaucoma in CxWT littermates produced a significant loss of both BRN3A-positive RGCs and dACs in the GCL, similar to that observed in microbead-injected C57BL/6 mice (Figure 3, I and J). Conversely, we observed no significant loss of cells in the GCL of microbead-injected $\mathrm{C} \times 36^{-/}$mice (Figure 3, I and $\mathrm{K}$ ). We found no further improvement in the protection of RGCs or dACs in retinas from microbead-injected eyes of $C \times 36^{--} \mathrm{Cx} 45^{--}$as compared with that found in $\mathrm{C} \times 36^{-/-}$mice, consistent with aforementioned comparisons of the 2 knockout mouse strains (Figure 3K).
Blockade or ablation of GJs prevents AC loss in experimental glaucoma. Our recent finding that $\mathrm{dACs}$ are lost in microbead-injected control mice (22) is consistent with studies showing that certain subpopulations of ACs are injured in animal models of glaucoma $(20,21)$. Moreover, ACs that are coupled to RGCs via GJs, including GABAergic and calretinin-positive (CR-positive) cells, are particularly vulnerable, suggesting that their loss in glaucoma reflects secondary degeneration via the bystander effect (22). We therefore extended our study to determine whether blockade of GJs could offer significant protection to these vulnerable AC subpopulations in glaucomatous eyes. Consistent with previous reports $(22,33)$, antibodies against GABA and CR labeled neuronal cell bodies in the INL and GCL of control retinas with dendritic processes forming characteristic bands in the IPL (Figure 4, A and F). Eight weeks after initial microbead injection we observed a significant reduction in the number of both GABA- and CR-positive cells in the INL and GCL of CxWT mice, which was accompanied by marked changes in the density of labeled dendrites in the IPL (Figure 4, B, G, K, and M). However, blockade of GJs with MFA provided significant protection of both GABA- and CR-positive neurons in terms of cell number and the preservation of normal dendritic ramification in the IPL (Figure 4, C, H, K, and M). Retinas from microbead-injected eyes of $C \times 36^{--}$mice also showed significant protection of GABA-positive (Figure 4, E and L) and CR-positive (Figure 4, J and N) AC subpopulations in terms of cell numbers and dendritic profiles in the IPL (Figure 4, D, I, L, and N). We found that deletion of both $C \times 36$ and $C x 45$ provided no further protection of ACs above that found with ablation of $C \times 36$ alone (Figure 4, L and N). Taken together with the comparisons of the protective effects of the 2 knockout strains described above, these data indicate that $\mathrm{Cx} 36$-expressing GJs play a principal role in the loss of both RGCs and ACs observed in experimental glaucoma.

Expression of $\mathrm{C} x 36$ is upregulated in experimental glaucoma. Consistent with previous studies $(12,34)$, strong punctate immunolabeling of $\mathrm{Cx} 36$, reflecting GJ loci, was observed in the inner portion of the IPL of C57Bl/6 mice, corresponding to the ON sublamina-b, with less labeling in the OFF sublamina-a (Figure $5 \mathrm{~A}$ ). In contrast, immunolabeling of $\mathrm{Cx} 45$ showed a more homogeneous pattern across both sublaminae of the IPL (Figure $5 F)$. Changes in the expression of connexins have been reported under different insult conditions $(12,17)$. Indeed, Western blot analysis indicated an increase in $\mathrm{Cx} 36$ protein in retinas at 8 weeks after initial microbead injection (Supplemental Figure 2). These data were extended by immunolabeling experiments, showing a significant increase in Cx36 puncta in the IPL, particularly in sublamina-a, of retinas at 8 weeks after initial microbead injection indicating an increase in Cx36-expressing GJs (Figure $5, \mathrm{~B}, \mathrm{D}$, and $\mathrm{E})$. The increase in the number of $\mathrm{Cx} 36$ puncta in the IPL began at 4 weeks after the initial microbead injection and gradually increased over the next 8-week period (Supplemental Figure 3). In contrast, no change in $\mathrm{Cx} 45$ immunolabeling was observed in microbead-injected retinas as compared with control levels (Figure 5, F-H).

Application of MFA to glaucomatous eyes prevented the upregulation of $\mathrm{Cx} 36$, maintaining expression levels at those seen under control conditions (Figure 5, C-E). These data are consistent with and extend our finding that Cx36-expressing GJs play 

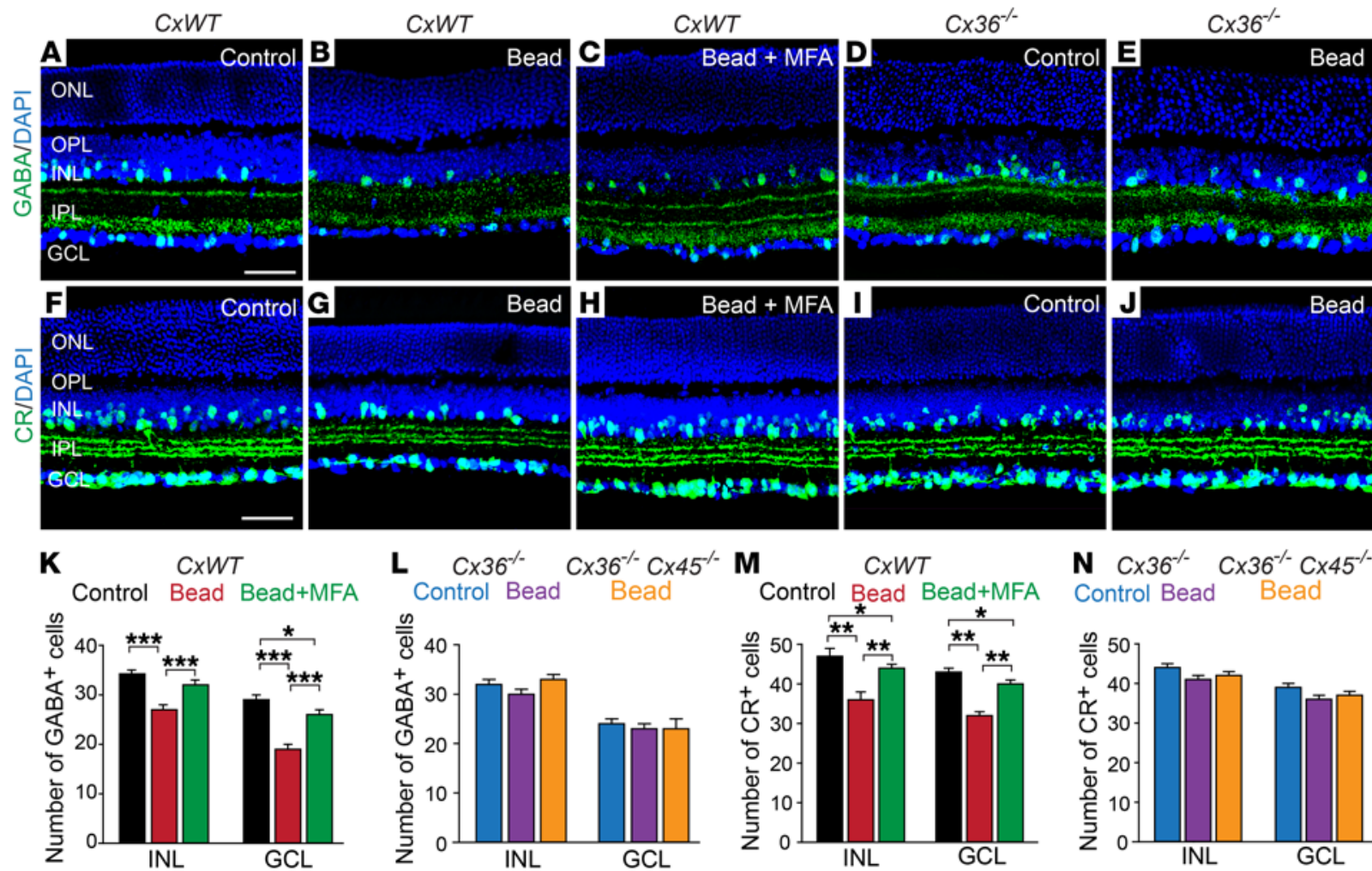

$\mathbf{N} \mathrm{C} \times 36^{-/-} \mathrm{C} \times 36^{-/-} \mathrm{C} \times 45^{-1-}$ Control Bead Bead

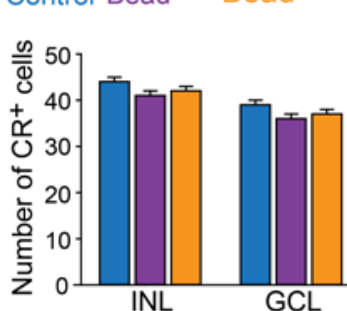

Figure 4. GJ blockade or ablation protects ACs in glaucomatous eyes. (A-C) Representative images of vertical sections of $C x W T$ mouse retinas immunostained for GABA and counterstained for DAPI under control conditions and 8 weeks after microbead injections with or without MFA treatment. Scale bar: $50 \mu \mathrm{m}$ for all panels. Z-stack: 5 sections, 2- $\mu \mathrm{m}$ steps. (D and E) Representative images of vertical sections of $C \times 36^{-/-}$mouse retinas immunostained for GABA and counterstained for DAPI under control conditions and 8 weeks after microbead injections. Z-stack: 5 sections, 2- $\mu \mathrm{m}$ steps. (F-H) Representative images of vertical sections of $C x W T$ mouse retinas immunostained for CR and counterstained for DAPI under control and glaucomatous conditions with or without MFA treatment. Scale bar: $50 \mu \mathrm{m}$ for all panels. Z-stack: 5 sections, 2- $\mu \mathrm{m}$ steps. (I and J) Representative images of vertical sections of $C \times 36^{-/-}$mouse retinas immunostained for $C R$ and counterstained for DAPI under control conditions and 8 weeks after microbead injections with or without MFA treatment. Z-stack: 5 sections, 2- $\mu$ m steps. (K) Quantification of the number of GABA-positive ACs in the INL and GCL of retinas from $C x W T$ mice under control and experimental conditions as presented in A-C ( $n=12$ retinas per group). (L) Quantification of the number of GABA-positive ACs in the INL and GCL of retinas from control and microbead-injected $C \times 36^{-/-}(n=10$ retinas per group) and $C \times 36^{-/-} C \times 45^{-/-}$mice ( $n=8$ retinas). (M) Quantification of the number of CR-positive cells in the INL and GCL of $C \times W T$ mouse retinas under conditions detailed in $\mathbf{F}-\mathbf{H}$ ( $n=10$ retinas per group). (N) Quantification of the number of CR-positive cells in the INL and GCL of retinas from control and microbead-injected $C \times 36^{-1-}(n=10$ retinas $)$ and $C \times 36^{-1-} C \times 45^{-/-}$mice $(n=8$ retinas $)$. Results are presented as mean $\pm \mathrm{SEM} .{ }^{*} P<0.05,{ }^{* *} P<0.01$, ${ }^{* *} P<0.001,1$-way ANOVA followed by Tukey's multiple comparisons test.

a principal role in progressive cell death in glaucoma, suggesting that upregulation of Cx36 may amplify the bystander effect and the resulting progressive neuron degeneration.

Cx36 ablation prevents optic nerve injury in experimental glaucoma. Since Cx36 is not expressed in the optic nerve (Supplemental Figure 4), we examined whether deletion of Cx36-expressing GJs in retina could offer protection of axons projecting centrally in glaucomatous animals. Cross sections obtained from the glial lamina area in mice, equivalent to the human lamina cribrosa, were immunolabeled with SMI32 and double labeled with the astrocyte marker GFAP. In optic nerves of control CxWT mice, GFAP was expressed in thick processes of astrocytes that formed the glial tubes, which ensheathed bundles of SMI32-positive axons creating a characteristic honeycomb mosaic (ref. 35 and Figure 6A). We found significant changes in the structure of the optic nerve at 8 weeks after the initial microbead injection. This included a disruption of the axonal bundles and the honeycomb pattern of astrocytic dendrites, which now appeared as short and thin processes that were disorganized (Figure 6B). Such morphological changes could account for the reduced area of GFAP labeling and the reduction of SMI32-positive axons in glaucomatous eyes as compared with values in control animals (Figure 6, E and G).

In contrast, we detected no significant change in the expression of GFAP and much smaller reduction of SMI32-positive axons, in comparison with $C x W T$ mice, in the optic nerve of microbead-injected eyes of $C x 36^{-/-}$mice, which maintained a normal honeycomb configuration (Figure 6, C, D, F, and H). Clearly, ablation of Cx36-expressing GJs in the retina preserved the structural integrity of the optic nerve in microbead-injected eyes.

CX36 ablation prevents attenuation of ERG and VEP components in glaucoma. Two components of the scotopic ERG, the positive scotopic threshold response (pSTR) and oscillatory potentials (OPs), were analyzed to evaluate the function of RGCs $(36,37)$ and ACs (38), respectively, in control and experimental animals. Eight weeks after microbead injection we observed significant reductions in the amplitude of the pSTR (Figure 7A) and the 

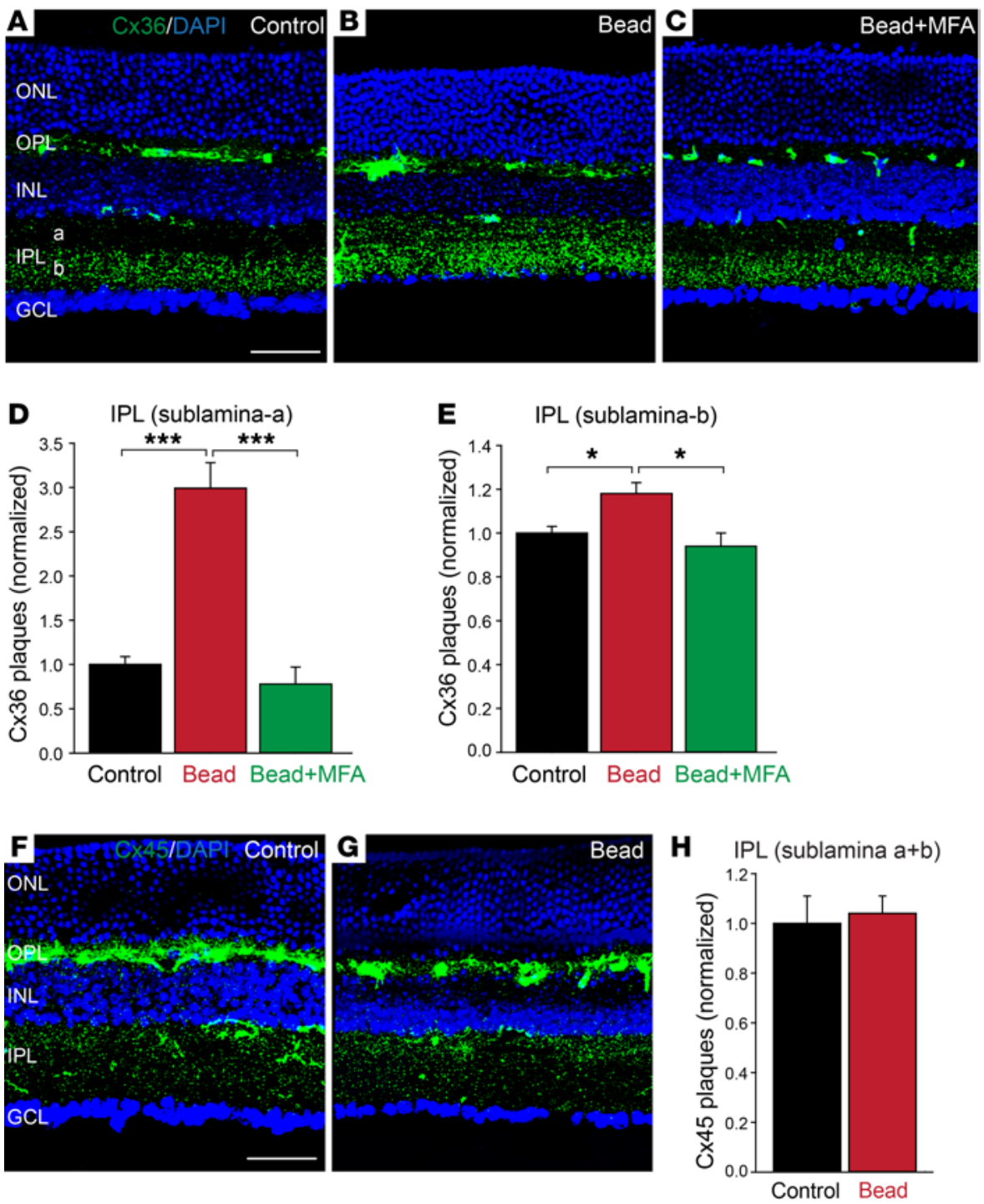

Figure 5. Expression of $\mathrm{Cx} 36$ is upregulated in experimental glaucoma. (A-C) Representative images of vertical sections of $\mathrm{C} 5 \mathrm{~B} \mathrm{BL} / 6$ mouse retinas immunostained for $\mathrm{C} \times 36$ and counterstained with DAPI under control condition and 8 weeks after microbead injection with or without MFA treatment. Scale bar: $50 \mu \mathrm{m}$ for all panels. (D and $\mathbf{E}$ ) Quantification of the ×x36 immunolabeled plaques revealed a significant upregulation in the IPL of the glaucomatous retina specifically in the sublamina-a. No significant change in Xx36 expression or distribution was observed in glaucomatous retinas of MFA-treated mice. Plaque counts were performed per $16 \times$ $300 \mu \mathrm{m}$ area for sublamina-a and $33 \times 300 \mu \mathrm{m}$ area for sublamina-b in at least 8 sections and averaged across retinas (5 retinas per group). (F and $\mathbf{G ) ~ R e p r e s e n t a t i v e ~ i m a g e s ~ o f ~ v e r t i c a l ~ r e t i n a l ~}$ sections immunostained for $[\times 45$ from control and glaucomatous mice. Scale bar: $50 \mu \mathrm{m}$ in both panels. (H) Quantification of the number of $\mathrm{Cx} 45$ immunoreactive plaques in the IPL of control and glaucomatous retinas ( $n=4$ retinas per group). Plaques were counted per $50 \times 300$ $\mu \mathrm{m}$ area covering both sublamina $(a+b)$ of the IPL. Z-stack: 5 sections, $0.7-\mu \mathrm{m}$ steps for all images. All data are presented as mean \pm SEM. ${ }^{*} P<0.05,{ }^{* *} P<0.001$, Student's $t$ test. summed amplitude of OPs (Figure 7D) in glaucomatous C57BL/6 mice as compared with those measured in control mice. Consistent with reports in human glaucoma (39) and a mouse model of diabetic retinopathy (40), the OPs in microbead-injected eyes also showed a significant shift in onset latency compared with controls (Figure 7D). Treatment of glaucomatous eyes with MFA preserved the amplitude of the pSTR as well as the amplitude and onset latency of the OPs at values comparable to those observed in control retinas (Figure 7, A and D). Animals treated with MFA alone under control condition did not show any significant difference in the amplitude of pSTR or OPs compared with untreated animals $(n=16$ eyes, $P>0.1)$. Analogously to C57BL/6 mice, injection of microbeads into the eyes of $C x W T$ mice produced a significant reduction in the amplitude of the pSTR (Figure 7B) and OPs and a prolonged latency of the OPs from stimulus onset (Figure $7 \mathrm{E})$. However, we observed no significant difference in the amplitudes of the pSTR and OP components of the ERG between control and microbead-injected $C \times 36^{-/-}$mice (Figure 7, $\mathrm{C}$ and F). The change in onset latency of OPs in microbead-injected $C x W T$ mice was also not observed in the microbead-injected $C \times 36^{-/-}$retinas.
To assess the propagation of signals to central visual structures, we measured the photopic visual evoked potentials (VEPs) in occipital cortex. The VEPs recorded in C57BL/6 mice 8 weeks after the initial microbead injection were significantly reduced in amplitude compared with those measured in control animals (Figure 7G). Treatment of C57BL/6 mice with MFA produced a small increase in the VEP amplitude compared with that recorded in untreated animals (Figure 7G). However, MFA preserved the VEP amplitude in microbead-injected mice to levels seen in control mice treated with MFA alone (Figure 7G). Similarly, glaucomatous $C x W T$ mice showed a marked reduction in VEP amplitude compared with control levels (Figure $7 \mathrm{H}$ ). The attenuation in VEP amplitude was not observed in microbead-injected $C \times 36^{-/-}$mice, indicating a significant functional protection of signal transmission from retina to central visual pathways (Figure 7I).

Cx36 ablation preserves normal visual behavior of glaucomatous animals. In the final series of experiments, we determined whether ablation of Cx36-expressing GJs could prevent the deterioration of visually guided behavior of mice associated with experimental glaucoma. Behavioral measures of spatial vision in $\mathrm{C} \times 36^{-/-}$mice 

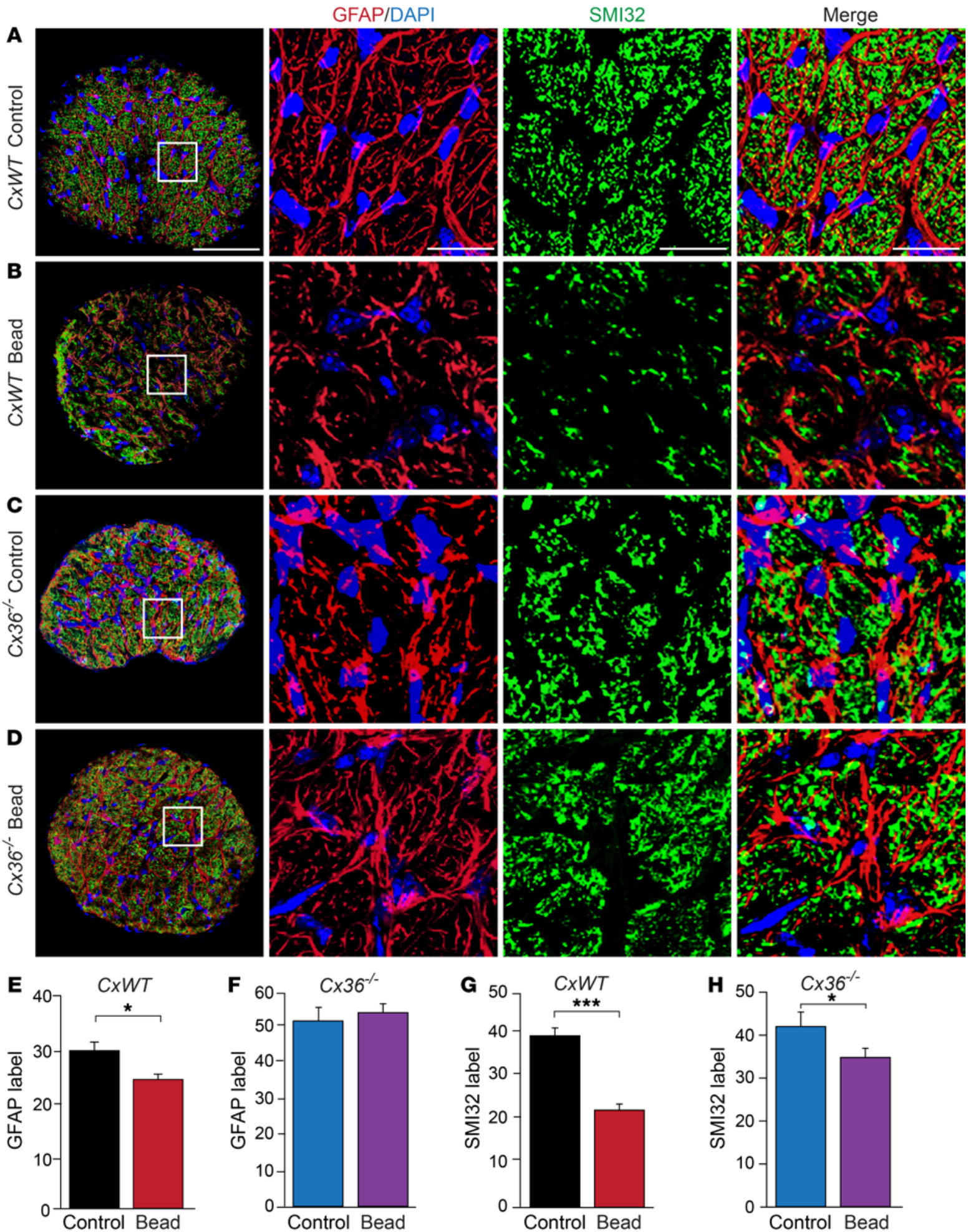

Figure 6. Ablation of neuronal Cx36 protects the optic nerve in experimental glaucoma. (A) Representative cross-sectional images of the glial lamina region of the optic nerve from control CXWT animals immunostained for GFAP, SMI32, and DAPI. Magnified images of the area outlined in the left panel show the immunolabeling pattern for GFAP and SMI32. Scale bars: $100 \mu \mathrm{m}$ for all panels in the left column and $25 \mu \mathrm{m}$ for panels in all other columns. (B) Representative cross-sectional images of the glial lamina region of the optic nerve from CxWT animals 8 weeks after initial microbead injection. Conventions are the same as in A. (C) Representative cross-sectional images of the glial lamina region of the optic nerve from $\left[\times 36^{-/-}\right.$mice under control conditions. Conventions are the same as in $\mathbf{A}$. (D) Representative cross-sectional images of the glial lamina region of the optic nerve from $C \times 36^{-/-}$animals 8 weeks after initial microbead injection. Conventions are the same as in A. (E) Quantification of GFAP labeling in the cross sections of optic nerves of $C x W T$ mice under control and glaucomatous conditions ( $n=5$ optic nerves per group). (F) Quantification of GFAP labeling in the cross sections of optic nerves of $C \times 36^{-/-}$mice under control and glaucomatous conditions ( $n=5$ optic nerves per group). (C) Quantification of SMI32 labeling in the cross sections of optic nerves of $C x W T$ mice under control and glaucomatous conditions ( $n=5$ optic nerves per group). (H) Quantification of SMI32 labeling in the cross sections of $C \times 36^{-/-}$mice under control and glaucomatous conditions ( $n=5$ optic nerves per group). Z-stack: 5 sections, $0.7-\mu \mathrm{m}$ steps for all images. Data are presented as mean \pm SEM. ${ }^{*} P<0.05,{ }^{* * *} P<0.001$, Student's $t$ test. 
and their $C x W T$ littermates were made under control conditions and then 8 weeks after microbead injection to assess changes in spatial acuity and contrast sensitivity. Mice were initially trained to perform a 2-alternate forced-choice task in a $\mathrm{Y}$ water maze to discriminate a test stimulus from a control gray screen $(41,42)$. Mice in each group were tested for contrast sensitivity to sinusoidal grating stimuli of $100 \%, 50 \%, 30 \%$, and $20 \%$ contrast, each at spatial frequencies of $0.1,0.3$, and 0.5 cycles per degree (cpd). Measures made 8 weeks after initial microbead injection indicated a small reduction in contrast sensitivity with borderline significance $(P=0.05-0.09)$ to gratings of $0.1 \mathrm{cpd}$ for both mouse strains (Figure 8A). However, contrast sensitivity to 0.3 - and 0.5 -cpd gratings was significantly reduced in microbead-injected $C x W T$ mice (Figure $8, \mathrm{~B}$ and $\mathrm{C}$ ). In contrast, we found no change in the contrast sensitivity of microbead-injected $C \times 36^{-/-}$mice for all gratings tested (Figure $8, \mathrm{~A}^{-} \mathrm{C}$ ). We next tested the spatial acuity of both $\mathrm{C} \times 36^{-/-}$and $\mathrm{C} x W T$ mice using 0.1-, 0.3-, and 0.5-cpd gratings at $100 \%$ contrast. Under control conditions, CxWT could readily discriminate all spatial gratings based on the $75 \%$ correct threshold criterion (Figure 8D). However, 8 weeks after microbead injection, the $C x W T$ mice showed significant deterioration in spatial acuity, as only the 0.1-cpd grating could be correctly discriminated (Figure 8D). In contrast, the spatial acuity of microbead-injected $\mathrm{C} \times 36^{-/-}$mice was indistinguishable from that seen under control conditions. Thus, ablation of $C x 36$ prevented the attenuation of the tested spatial vision parameters normally seen after induction of experimental glaucoma.

\section{Discussion}

Advancing visual dysfunction associated with glaucoma results from the progressive cell death and axonal loss in the retina and optic nerve $(3,20-22,43,44)$. While a variety of intracellular and extracellular cascades subserve cell loss in neurodegenerative diseases such as glaucoma (45), the mechanism(s) responsible for the progressive, secondary cell losses has remained unclear. Here we have demonstrated that intercellular communication mediated by neuronal GJs, consistent with bystander cell death (9-12), plays a critical role in the progressive loss of inner retinal neurons and optic nerve axons in experimental glaucoma. As a result, pharmacological blockade of retinal GJs with the nonselective inhibitor MFA offered significant protection of the retina, largely preventing the thinning of the nuclear and plexiform layers and the reactive gliosis linked to overall structural damage. Moreover, GJ blockade produced a marked increase in the survivability of RGCs and ACs in glaucomatous eyes, with preservation of cell numbers and soma-dendritic architecture to those observed in control retinas. The finding that blockade of GJs resulted in nearly complete protection of ACs is consistent with recent work showing that ACs coupled to RGCs via GJs are highly vulnerable in glaucomatous retinas, whereas those not coupled are largely undamaged (22). These data suggest that AC loss in glaucoma occurs secondary to RGC loss via the GJ-meditated bystander effect. This preservation of inner retinal neurons resulted in a corresponding protection of retinal function as indicated by preservation of pSTR and OP components of the ERG at control levels.

It has been shown that different cohorts of GJs, based on their connexin makeup, mediate secondary cell death in the retina under different insult conditions (12). In our model of experimen- tal glaucoma, we found that genetic ablation of $C \times 36$ produced significant protection of retina structure and function. Interestingly, ablation of both $C \times 36$ and $C \times 45$, which are expressed by most, if not all, GJs in the inner retina, produced no additional protection compared with knockout of $C \times 36$ alone. In addition, we found a significant upregulation of $\mathrm{Cx} 36$, but not $\mathrm{Cx} 45$, protein in the OFF sublamina-a of the IPL in glaucomatous retinas, suggesting an amplification of the bystander effect, which was prevented by GJ blockade with MFA. Interestingly, recent studies have demonstrated that OFF $\alpha$-RGCs, which ramify their processes in the OFF sublamina of the IPL, are initially more vulnerable to glaucomatous insult than ON $\alpha$-RGCs (46-48). Overall, these results indicate that Cx36-expressing GJs play a principal role in secondary cell loss in glaucoma and thus form a selective target for neuroprotection.

The loss of RGCs in human and animal models of glaucoma often shows territorial heterogeneity $(45,49)$. An important question then is whether the bystander effect subserved by gap junctional coupling can provide for such a pattern of cell death. Although tracer coupling through GJs usually shows a symmetrical configuration (50), the extent and strength of coupling of Cx36-expressing GJs in both inferior olive (51) and retina (52) can exhibit marked heterogeneity. Thus, while it remains unclear whether the initial cell loss in glaucoma shows territoriality, the variability in GJ conductances could provide for or extend heterogeneous cell loss through the bystander mechanism.

In addition to neuronal GJs, many non-neuronal cells in the retina and optic nerve, including astrocytes, Müller cells, and microglia, also maintain GJs. However, these glial GJs express $\mathrm{Cx} 43$, indicating a different subunit structure from those expressed by neurons. As glial cells play a key role in cell homeostasis, Cx43expressing GJs have been implicated in the brain's response and overall susceptibility to ischemia, inflammation, and injury (53). In models of retinal ischemia, blockade of $\mathrm{Cx} 43 \mathrm{GJs}$ or prevention of $\mathrm{Cx} 43$ protein upregulation reduced overall cell death and injury in the retina and optic nerve $(17,54)$. Interestingly, $\mathrm{Cx} 43$ protein in retina is upregulated following optic nerve injury, and it has thus been suggested that glial GJs or hemichannels may play a role in neuronal loss associated with glaucoma (55). However, a direct link between $\mathrm{Cx} 43$ and the pathogenesis of neuronal death in glaucoma has not yet been established.

In contrast, the present results indicate a key role in glaucoma for bystander cell death mediated by Cx36-expressing neuronal GJs. In addition to the structural and functional protection of retina, we found that ablation of $\mathrm{Cx} 36$ protein produced significant protection of optic nerve structure, including RGC axons. The maintenance of optic nerve integrity in microbead-injected $C \times 36^{-/-}$ mice was supported by the conservation of the cortical VEP as well as spatial and contrast sensitivity at control levels, indicating that visual signals were effectively propagated from retina to brain. The optic nerve has been recognized as the initial site of damage leading to death of RGCs in glaucoma, classifying it as an axogenic disease $(1,2)$. Emerging evidence, however, indicates that morphological and functional changes in RGCs may occur prior to the optic nerve degeneration (46-48). Interestingly, the protection afforded to the optic nerve in microbead-injected $\mathrm{C} \times 36^{-/-}$animals is intriguing, in that $\mathrm{Cx} 36$ protein is not expressed there, suggest- 
A Control Bead Bead+MFA
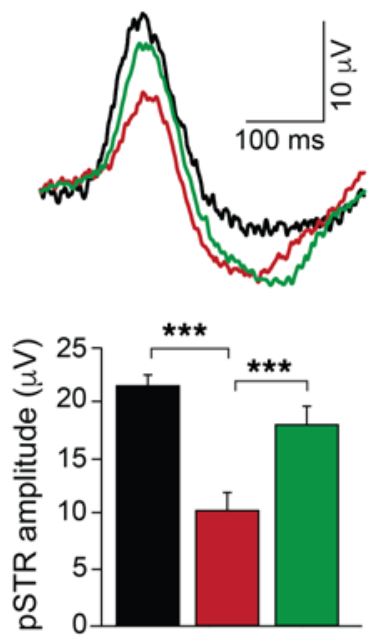

D Control Bead Bead+MFA
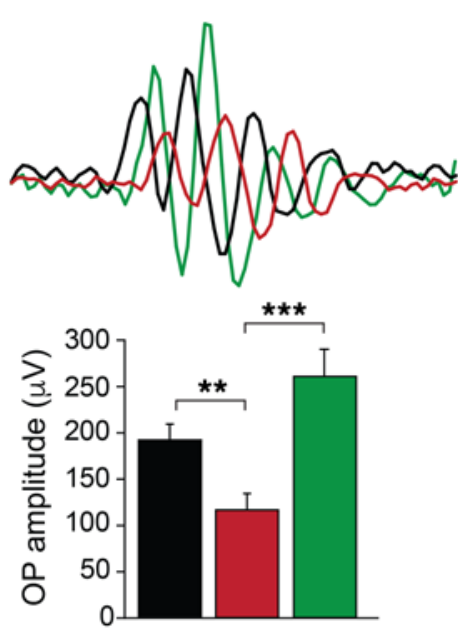

G
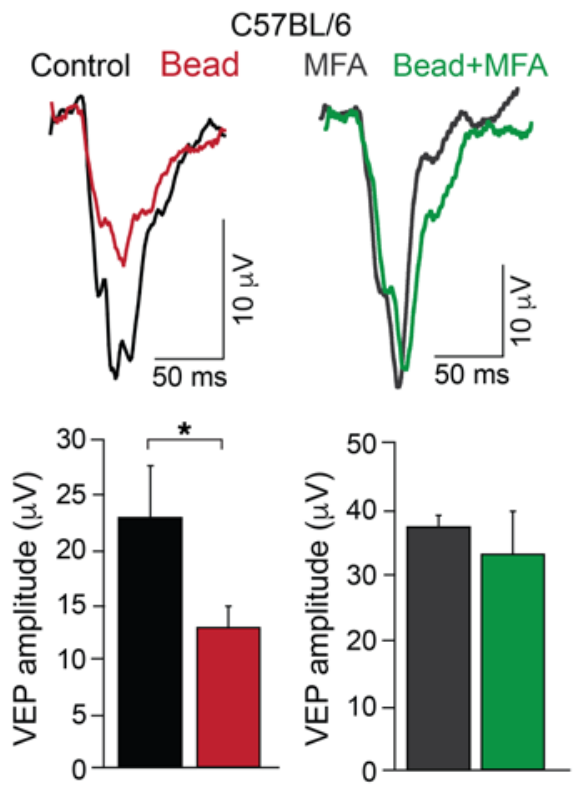

B

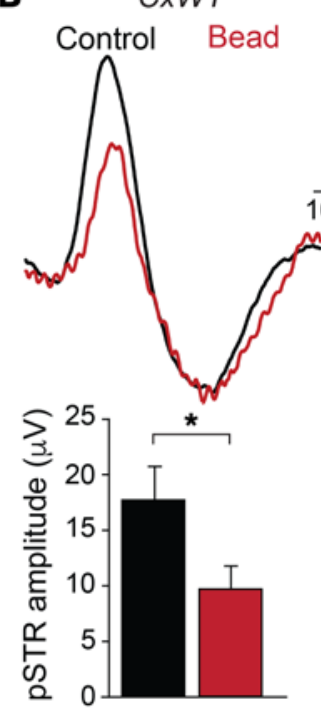

E $\quad C x W T$
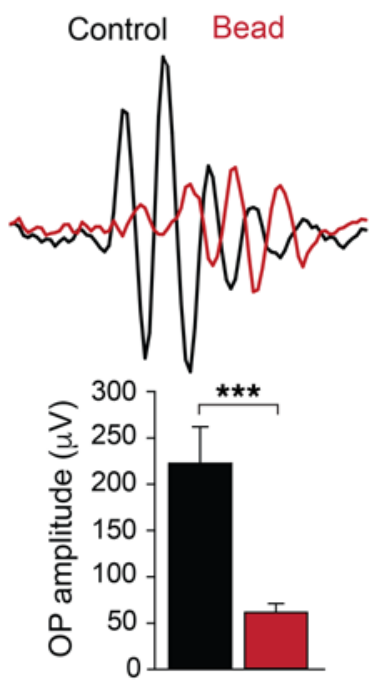

H

H CxWT Control Bead
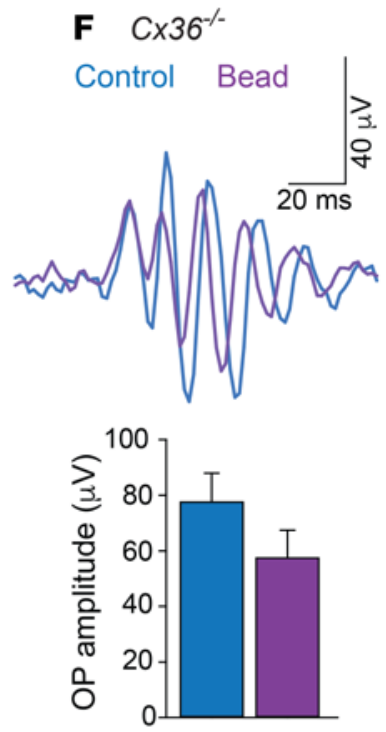
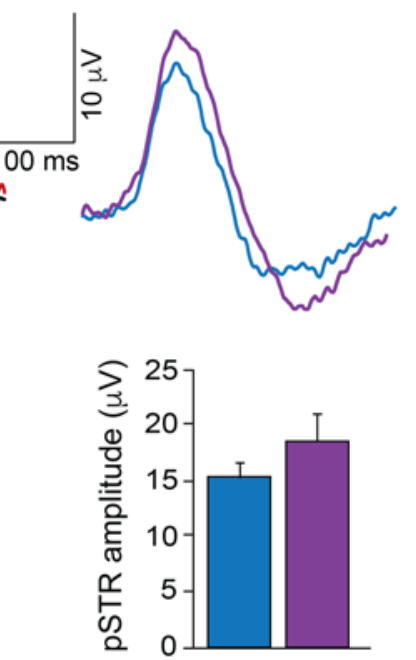

F $\mathrm{C} \times 36^{-/}$

ms
C $\mathrm{C} \times 36^{-/}$

Control Bead

Figure 7. Blockade of neuronal gap junctions preserves retina and optic nerve function in glaucoma. (A) pSTR of scotopic ERG from C57BL/6 mice under control conditions and 8 weeks after initial microbead injection with and without MFA. Histogram of the PSTR peak amplitude under different conditions ( $n=17$ eyes per condition). (B) pSTR from CXWT mice. Histogram of pSTR peak amplitude changes under different conditions ( $n=9$ eyes per condition). (c) pSTR from $C \times 36^{-1}$ mice. Histogram of pSTR peak amplitude changes under different conditions ( $n=15$ eyes per condition). Light intensity $=-4.3 \log \mathrm{scot}$. $\mathrm{cd} \cdot \mathrm{s} / \mathrm{m}^{2}$ for panels A-C. (D) OPs of the scotopic ERG from C57BL/6 mice. Histogram of OP summed amplitude under different conditions ( $n=14$ eyes per condition). (E) OPs of CxWT mice. Histogram of OP summed amplitude from control ( $n=10$ eyes) and glaucomatous mice ( $n=8$ eyes). (F) OPs recorded in $\mathrm{C} \times 36^{-/}$mice. Histogram of OP summed amplitude from control ( $n=15$ eyes) and glaucomatous ( $n=14$ eyes) mice. Light intensity $=-0.4 \log$ scot. $\mathrm{cd} \cdot \mathrm{s} / \mathrm{m}^{2}$ for panels D-F. (C) Photopic VEPs from control and glaucomatous [57BL/6 mice. Histogram of VEP peak amplitude in control ( $n=9$ mice) and glaucomatous mice ( $n=7$ mice). (H) VEPs from CXWT mice. Histogram of VEP peak amplitude under different conditions ( $n=5$ mice per condition). (I) VEPs from $\left[\times 36^{-/}\right.$mice. Histogram of VEP peak amplitude under different conditions ( $n=5$ mice per condition). Light intensity $=1.85 \mathrm{log} \mathrm{cd} \cdot \mathrm{s} / \mathrm{m}^{2}$ for panels G-I. Data presented as mean \pm SEM. ${ }^{*} P<0.05,{ }^{* *} P<0.01,{ }^{* * *} P$ $<0.001$, Student's $t$-test for B, C, E-I and 1-way ANOVA followed by Tukey's multiple comparison test for $\mathbf{A}$ and $\mathbf{D}$. 

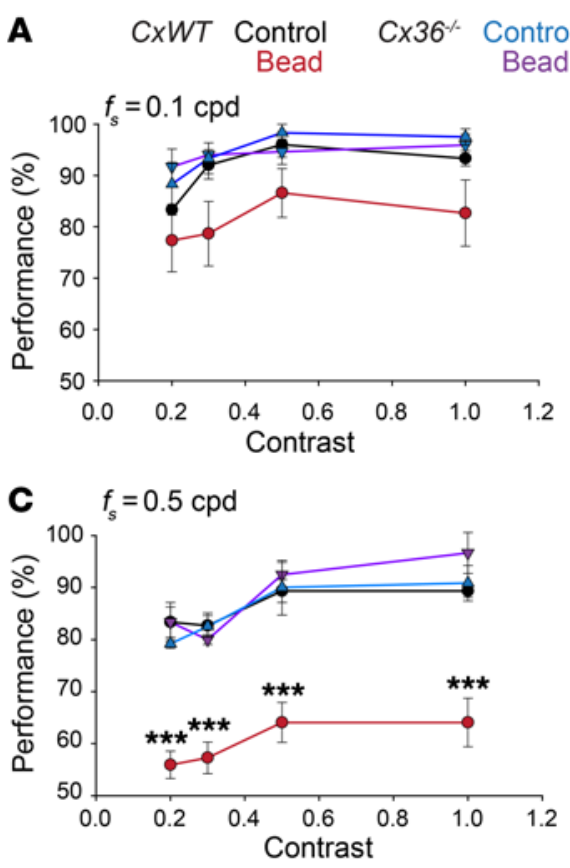

B
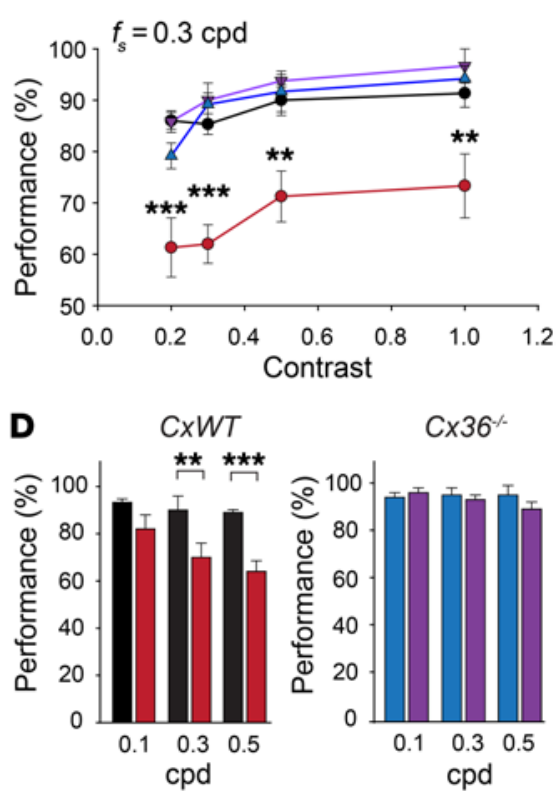

Figure 8. Ablation of GJs prevents a decline in behavioral measures of spatial vision in glaucomatous eyes. (A-C) Performance of CXWT mice ( $n=5$ animals per condition), and $C \times 36^{-/-}$mice ( $n=4$ animals per condition) under control and glaucomatous (8 weeks after initial bead injection) conditions, on a discrimination task between sinusoidal grating and gray visual stimuli. Grating frequencies $\left(f_{\mathrm{s}}\right)$ were $0.1,0.3$, and $0.5 \mathrm{cpd}$, presented at contrasts ranging from $20 \%$ to $100 \%$. (D) Changes in spatial acuity, based on a threshold performance of $75 \%$, calculated from data in A obtained at $100 \%$ contrast. Induction of glaucoma produced a decline in discrimination performance of $C x W T$ mice for all gratings, indicating reduced spatial acuity. In contrast, there was no change in the discrimination performance of microbead-injected $\left[\times 36^{-/-}\right.$ mice, indicating a preservation of spatial acuity. All data are presented as mean \pm SEM. ${ }^{* *} P<$ $0.01,{ }^{* *} P<0.001,1$-way ANOVA followed by Tukey's multiple comparisons test. ing that the pathogenesis of the optic nerve and retina, the latter where Cx36 is localized, must be mechanistically linked.

Taken together with results of previous studies $(6-8,22)$, our findings suggest a novel framework for understanding and thereby preventing the progressive damage associated with glaucoma. Whether the damage from elevated IOP is initiated in the optic nerve with axonal loss leading to retrograde somatic injury or begins with somatic RGC loss in the retina, the result is the death of the most vulnerable RGCs. The residual loss of RGCs and optic nerve axons that we found after GJ blockade or $\mathrm{Cx} 36$ ablation in glaucomatous eyes likely reflects this primary injury. It is widely believed that the primary RGC loss subsequently promotes secondary degeneration of surrounding healthy cells $(2,7,8,18,45)$. The present findings indicate that the GJ-mediated bystander effect plays a critical role in the spread of injury and secondary cell death across the retina. This injury also results in an upregulation of Cx36, which strengthens GJ communication, thereby amplifying and extending secondary cell loss. In turn, this secondary degeneration or bystander cell death results in anterograde degeneration of RGC axons, thereby expanding the damage to the optic nerve. As a result, the secondary cell loss via the bystander effect may ultimately be responsible for the majority of cellular and axonal loss over time.

Our results thus reveal neuronal GJs, specifically those expressing Cx36, as potential targets for novel neuroprotective therapies to prevent the progressive retinal damage and visual deficits associated with glaucoma. In our experimental paradigm, blockade of GJs or connexin deletion occurred prior to induction of glaucoma, and so it will be interesting to see whether intervention after retinal injury occurs can significantly reduce progressive cell death. Finally, deletion of $C x 36$ in mice produces a significant reduction of RGC scotopic responses, but photopic responses appear unaffected $(56,57)$, although some changes in ERG components have been reported under bright light conditions (58). Clearly, titration of potential drugs that can selectively block Cx36-expressing GJs will be necessary to establish a therapeutic dose in humans, which maximizes neuroprotective actions while minimizing any deleterious effects of GJ obstruction on vision.

\section{Methods}

Animals. Experiments were performed on either C57BL/6 mice or connexin knockout ( $\mathrm{C} \times 36^{-/}$and $\mathrm{C} \times 36^{-/} \mathrm{C} \times 45^{-/}$double-knockout) mice and their wild-type $(C x W T)$ littermates. The $C x 36^{-/-}$mice and littermates were derived from $\mathrm{F}_{2} \mathrm{C} 57 \mathrm{BL} / 6-129 \mathrm{SvEv}$ mixed-background litters (56). To create $C x 36^{--} C x 45^{-/}$mice, we used $C x 45^{--}$mice (59), which were first generated in David Paul's laboratory (Harvard Medical School, Boston, Massachusetts, USA) by crossing of $C \times 45^{f / f l}$ mice with mice expressing Cre recombinase under control of the neuron-directed nestin promoter to yield $C x 45^{f / f l}$ nestin-Cre mice. These were then bred with $C x 36^{-/-}$or $C x 36^{+/-}$mice to yield $C x 45^{f / f l} C \times 36^{-/-}$ nestin-Cre mice (mice lacking both $\mathrm{Cx} 36$ and $\mathrm{Cx} 45$ ). The C57BL/6 mice were originally obtained from The Jackson Laboratory and subsequently bred in the animal care facility at the College of Optometry, State University of New York. Animals were kept under a 12:12-hour ambient light cycle and fed ad libitum. For all experiments, animals were selected randomly within each group. All the experiments were performed on 3- to 4-month-old mice of either sex.

Induction of elevated IOP by microbead injection. The intraocular pressure (IOP) was elevated by injection of $10-\mu \mathrm{m}$-diameter polystyrene microbeads (Invitrogen) into the anterior chamber as previously described (24). The intracameral injections were performed unilaterally with $2 \mu$ of microbead suspension (containing $7.2 \times 10^{6}$ beads) using a glass micropipette connected to a microsyringe. The cornea was gently punctured using a 30-gauge needle before intracameral injections. An equivalent volume of PBS was injected into the contralateral eyes to provide control (sham) measurements. A second microbead injection was performed during the fourth week, which maintained elevated IOP for at least 8 weeks. All injections were performed on 
animals anesthetized with an i.p. injection of ketamine/xylazine mixture and topical application of $0.5 \%$ proparacaine. Enucleations were performed at 8 weeks after the initial microbead injection, and retinas were assessed for gross structural and cellular damage.

IOP measurements. Measurements of IOP were made using a tonometer (TonoLab; Colonial Medical Supply) as previously described (25). The IOP measurements were made initially within 1-3 minutes after anesthetizing of the mice with an i.p. injection of the ketamine/xylazine mixture and topical application of $0.5 \%$ proparacaine. Subsequent measurements were performed weekly between 10 am and $12 \mathrm{pm}$, to minimize the effect of diurnal IOP variation. For each weekly IOP measurement, 6 readings were averaged for each eye.

Pharmacological blockade of GJs. The GJ blocker meclofenamic acid (MFA; Sigma-Aldrich) was delivered to C57BL/6 or CxWT mice via subcutaneous osmotic minipumps (model 2004; Alzet). In some early experiments we also administered MFA by intravitreal injection $(2 \mu \mathrm{l}$ of 500- $\mu \mathrm{M}$ solution). The 2 methods of MFA administration produced qualitatively similar results in terms of protection of neurons and prevention of retinal injury (compare Figure 3, E and G, and Supplemental Figure 5). However, unlike humans, the lens in the mouse eye occupies most of the globe. Thus, multiple intravitreal injections carried out weekly often could not avoid damage to the lens, resulting in a cataract that could compromise light stimulation used in ERG and VEP recordings and behavioral experiments. Therefore, all MFA was delivered by osmotic minipumps in all experiments reported here. To implant the minipumps, mice were anesthetized under surgical isoflurane anesthesia and placed on a heating pad. The minipumps were filled with an MFA solution and inserted subcutaneously on the dorsum by an incision 1.5 times the diameter of the pump, and the wound was closed with suture (nylon 6-0). Pumps were implanted 1 day before microbead injection and replaced at 4 weeks. After 8 weeks, ERG and VEP recordings were performed, followed by histochemical experiments. To determine the optimum dosage of MFA at which it blocks the GJ between RGCs and ACs, we administered different doses of MFA $(5,10$, and $20 \mathrm{mg} / \mathrm{kg} / \mathrm{d})$. After 1 week, RGCs in control and MFA-treated eyes were retrogradely labeled through the optic nerve cut (as described below) with the GJ-permeant tracer Neurobiotin (NB; Vector Laboratories), and coupling was evaluated by the presence of NB-labeled ACs in the INL $(12,60)$. We found that MFA at doses of 5 and $10 \mathrm{mg} / \mathrm{kg} / \mathrm{d}$ had little effect on gap junctional coupling, whereas at a dose of $20 \mathrm{mg} / \mathrm{kg} / \mathrm{d}$ MFA effectively blocked the coupling between RGCs and ACs as evidenced by the absence of NB-labeled ACs in the INL (Supplemental Figure 6, A-C). The effectiveness of this approach to assess coupling was tested by evaluation of AC labeling in eyes of $C \times 36^{-/-}$mice retrogradely labeled with NB. Similar to the effect of MFA dosed at $20 \mathrm{mg} / \mathrm{kg} / \mathrm{d}$, no NB-labeled ACs were observed in the INL of retinas from $C x 36^{-/-}$mice (Supplemental Figure 6D).

Retrograde labeling of retinal neurons. The method for retrograde labeling of RGCs has been reported previously (12). Briefly, isolated globes with attached optic nerves were submersed in oxygenated Ames medium, and a drop of NB (4\% in $0.1 \mathrm{M}$ Tris buffer) was applied to the optic nerve cut for 40 minutes. The cornea and lens were removed, and the remaining retina eyecups were washed in oxygenated Ames medium for 1 hour. The tissues were then subjected to histological analysis as described below.

Immunohistochemistry. The immunohistochemical methods have been described previously (12). Briefly, the eyecups were fixed with $4 \%$ paraformaldehyde in 0.1 M PBS, pH 7.4, for 30 minutes at room tem- perature, and some were used for whole-mount preparation while others were cryoprotected in $30 \%$ sucrose, embedded in tissue freezing medium (72592; Electron Microscopy Science), and cut as 10- $\mu$ m-thick frozen sections. Vertical retinal sections or retinal whole mounts were blocked in 0.1 M PBS containing 10\% normal donkey serum (NDS), $1 \%$ BSA, and 0.5\% Triton X-100 for 1 hour. The tissues were then incubated with primary antibodies diluted in 0.1 M PBS containing 3\% NDS, 1\% BSA, and $0.1 \%$ Triton X-100 overnight (sections) or for 48 hours (retinal whole mount) at $4^{\circ} \mathrm{C}$. After an extensive washing in $0.1 \mathrm{M} \mathrm{PBS}$, tissues were incubated for 2-4 hours in secondary antibodies. Tissues were then mounted in Vectashield media with DAPI (H-1200; Vector Laboratories). Images of immunolabeled tissues were taken by Olympus FV1200 MPE confocal microscope (Olympus) with $\times 20$ or $\times 40$ (oil immersion) objectives. High-resolution (1,024 × 1,024 pixels) Z-stack images were taken using step size of 0.7-2.0 $\mu \mathrm{m}$, compiled to a single plane, and analyzed quantitatively. The brightness and contrast of micrographs were adjusted using Photoshop CS6 (Adobe). The primary antibodies used were anti-GFAP (1:1,000, RA22101; Neuromics), anti-BRN3A (1:500, sc-31984; Santa Cruz Biotechnology), anti-GABA (1:500, PC213L; EMD Millipore), anti-calretinin (1:1,000, PA5-16681; Invitrogen), SMI32 (1:2,000, SMI32R-100; Covance), anti-Cx36 (1:200, MAB3045; EMD Millipore), and anti-Cx45 (1:200, SC374354; Santa Cruz Biotechnology). The secondary antibodies used were donkey anti-rabbit, -mouse, and -goat conjugated with Alexa Fluor 488, 594, and 633, respectively (1:200; A-21206, R-37115, A-21082; Life Technologies). NB was visualized with Alexa Fluor 488/594-conjugated streptavidin (1:200, S32356; Invitrogen).

Assessment of retinal injury and neuronal death. The detailed methods to assess retinal injury and neuronal loss have been described previously (12). It has been shown that neuronal death in the mouse glaucoma model can vary by sector eccentricity (61), and so all measures were made from the midperipheral regions, 1.5-2.0 $\mathrm{mm}$ from the optic disk. However, no variability occurs between retinal quadrants (61), and so measurements made in midperipheral regions within different quadrants were summed and averaged. The total number of cells in GCL was computed using DAPI labeling of cell nuclei, and RGCs were labeled with BRN3A. BRN3A has been shown to label 86\%-92\% of RGCs in the rodent retina $(31,62)$. Vertically sectioned retina cell counts of DAPI- and BRN3A-labeled cells were performed manually per unit length of $630 \mu \mathrm{m}$ within the midperipheral regions. The number of RGCs was then subtracted from the total number of cells in the GCL to derive the number of dACs in the GCL. For retinal whole mounts, BRN3A-positive RGCs were counted within $600 \times 600 \mu \mathrm{m}$ square area in each of the 4 retinal quadrants from the midperipheral region, and numbers were averaged for at least 3 retinas per each control and experimental condition. Cells in retinal whole mounts were counted using particle analysis in ImageJ (NIH). To measure a thickness of individual retinal layers, confocal images of DAPI-stained vertical sections from midperipheral retina were acquired at $\times 40$ magnification, and measurements were performed at similar distance $(\sim 2$ $\mathrm{mm}$ ) from the optic nerve head using Fluoview software (Olympus). To assess gliosis caused by high IOP, we utilized Image J analysis software (NIH), and counted the number of pixels with GFAP label above background in images obtained from individual retinal layers GCL, IPL, INL, and OPL of retinal whole mounts. The number of GFAP-positive pixels was then divided by the total number of pixels in the image and presented as a percentage of GFAP-covered area. Three square 
areas of $300 \times 300 \mu \mathrm{m}$ in each quadrant (midperipheral region) were selected for analysis, and values were averaged across at least 3 control and 3 experimental retinas for each protocol. This parameter was independent of differences in the intensity of label within or across retinas. Similar measurements were performed to evaluate the SMI32-positive axons in the whole mounts from control and experimental retinas. The thickness of the individual retinal layers was measured in $\times 40$ magnified images of vertical retinal sections selected from the midperipheral region and stained with DAPI using Olympus confocal microscope and Fluoview FV1000 software. All data were imported into Sigmaplot software (Systat Software), and histograms were constructed.

Optic nerve preparation and immunostaining. Globes attached with optic nerves were isolated and then dissected approximately $1 \mathrm{~mm}$ anterior to the optic nerve head. The posterior part of the eye with optic nerve was fixed with $4 \%$ paraformaldehyde and cryoprotected as described above, and then cross sections of 8-10 $\mu \mathrm{m}$ were cut through the glial lamina region. Cross sections were immunostained for GFAP and SMI32, and mounted on a glass slide with Vectashield medium containing the nuclear stain DAPI (Vector Laboratories). To assess the axonal density, the percentage of cross-section area covered by SMI32-positive axons was measured in 8-10 cross sections per optic nerve and averaged for total number of optic nerves in each control and experimental condition.

Western blot. Retinal tissue samples from control and microbeadinjected eyes were collected and homogenized using an electric homogenizer in lysis buffer containing 0.25 M Tris, $1.92 \mathrm{M}$ glycine, and $1 \%$ SDS in PBS with Mini Complete Protease Inhibitor (Thermo Fisher Scientific) and stored at $-80^{\circ} \mathrm{C}$. The protein concentration was quantified using the Micro BCA assay (Thermo Fisher Scientific) according to the manufacturer's instructions. A 40- $\mathrm{g}$ protein sample from each group was diluted in loading buffer $(60 \mathrm{mM}$ Tris- $\mathrm{HCl}$, pH 6.8, 5\% $\beta$-mercaptoethanol, 2\% SDS, $10 \%$ glycerol, $0.025 \%$ bromophenol blue) and heated at $95^{\circ} \mathrm{C}$ for 5 minutes. The samples along with molecular weight markers were electrophoresed on $12 \%$ SDSpolyacrylamide gel and transferred onto a PVDF membrane (Thermo Fisher Scientific). The membrane was then blocked with Amersham ECL Prime Blocking Reagent (GE Healthcare Life Sciences) 1.5\% in 1× PBS and 0.1\% Tween-20 (PBST) for 1 hour at room temperature. The membrane was incubated overnight at $4^{\circ} \mathrm{C}$ with a mouse anti-connexin 36/35 monoclonal primary antibody (1:250, catalog MAB3045; EMD Millipore) diluted in blocking buffer. After 4-5 washes with PBST, each for 5 minutes, the membrane was incubated for 2 hours at room temperature with an anti-mouse secondary antibody conjugated to HRP (1:10,000, catalog sc-2371; Santa Cruz Biotechnology), which was diluted in blocking buffer. After PBST washing, the proteins were detected using an ECL detection system (SuperSignal West Femto Maximum Sensitive Substrate; Thermo Fisher Scientific) according to the manufacturer's instructions. For internal control, membrane was stripped and reprobed for $\beta$-actin mouse monoclonal antibody (1:200, catalog sc-47778; Santa Cruz Biotechnology) using the same procedure as described above. The mean band intensities were determined using Image J and normalized against $\beta$-actin band as loading control.

ERG and VEPs. Scotopic ERGs were recorded as described previously (22). Mice were dark-adapted overnight and anesthetized with ketamine $(70 \mathrm{mg} / \mathrm{kg})$ and xylazine $(7 \mathrm{mg} / \mathrm{kg})$. Pupils were dilated with $2.5 \%$ phenylephrine hydrochloride (17478-200-12; Akorn) and accommodation blocked with $1 \%$ tropicamide (17478-101-12; Akorn) solution, under dim red light, and $1 \%$ methyl cellulose was used to moisten the cornea. Body temperature was maintained around $37^{\circ} \mathrm{C}$ with an electric heating pad. Platinum electrodes were placed on the corneal surface of both eyes to record ERGs; needle reference and ground electrodes were inserted into the cheek and the skin in the back of the neck, respectively. The ERG responses were recorded from both eyes simultaneously. Visual stimuli consisted of brief $(<5 \mathrm{~ms})$ white Ganzfeld flashes ranging between -6.7 and $2.0 \log \mathrm{scot} . \mathrm{cd} \cdot \mathrm{s} / \mathrm{m}^{2}$ (scotopic candela seconds per meter squared), produced by an array of light-emitting diodes (Espion ColorDome Stimulator; Diagnosys). Responses were averaged over 40-50 trials for weak stimuli and fewer trials for stronger stimuli. Signals were amplified, filtered $(1-300 \mathrm{~Hz})$, and digitized at $1 \mathrm{kHz}$ with a resolution of $0.1 \mu \mathrm{V}$. The STR was elicited in the intensity range of -4.9 to $-4.0 \log \mathrm{scot}$. $\mathrm{cd} \cdot \mathrm{s} / \mathrm{m}^{2}$, and the amplitude of positive STR (pSTR) was measured from baseline. Responses in the intensity range of -0.4 to $2.0 \mathrm{log} \mathrm{scot}$. $\mathrm{cd} \cdot \mathrm{s} / \mathrm{m}^{2}$ were band pass filtered $(70-300 \mathrm{~Hz})$ to extract the oscillatory potentials (OPs). The OP amplitude was expressed as the sum of the first 5 OPs (OP1-OP5) measured from trough to peak, and the OP timing was expressed as the sum of the OP1-OP5 peak times. Visual evoked potentials (VEPs), which reflect activity of the visual cortex, were recorded under photopic conditions with a $5-\mathrm{ms}$ flash of $1.85 \mathrm{log} \mathrm{cd} \cdot \mathrm{s} / \mathrm{m}^{2}$ stimulus strength following 10 minutes of light adaptation (63). A needle scalp electrode placed under the skin close to midline near the visual cortex served as the recording electrode with the same reference and ground as for the ERGs. To reduce noise levels in recordings, 200-250 individual sweeps were averaged and recorded.

Behavioralmeasures of spatial vision. The vision of mice was assessed with a behavioral assay performed in a commercially available $\mathrm{Y}$ water maze (San Diego Instruments) with sectional dimensions of $15 \times 3 \times 6$ inches $(1 \times w \times h)$. Each of the 2 split arms of the $\mathrm{Y}$ water maze faced a computer monitor, both of which were calibrated for equal luminance. Each monitor pseudorandomly displayed either a gray screen or spatial sine wave grating (64) encoded in Matlab (Mathworks) and Psychtoolbox (65) that respectively served as the negative and the positive stimuli. We found that animals in which subcutaneous minipumps were inserted for MFA delivery had significant problems swimming the water maze, which compromised the ability to assess the visual behavioral tests. Furthermore, as described above, 7 weekly intravitreal injections of MFA often led to development of cataracts, due to accidental damage to the lens, that compromised animals' vision. We therefore limited the behavioral tests to $C x W T$ and $C x 36^{-/}$animals, which both navigated the water maze with similar proficiency. $C x W T$ and $C \times 36^{-1-}$ mice were trained to perform a 2-alternate forced-choice task $(41,42)$. Mice were continuously trained to swim to a platform placed in the arm facing the spatial gratings until the performance improved from $50 \%$ to $80 \%-90 \%$. After training, mice were evaluated for both spatial acuity and contrast sensitivity. Spatial acuity was assessed using gratings of $0.1,0.3$, and 0.5 cycles per degree (cpd) at $100 \%$ contrast. Contrast sensitivity was measured using the 0.1-, 0.3-, and 0.5-cpd gratings presented with contrast ranging from $20 \%$ to $100 \%$. A $75 \%$ accuracy was taken as the threshold of correct performance. After control values were obtained, a subset of animals received intracameral injections of microbeads bilaterally to induce glaucoma. The first 7 days (week 0) following the injection was allowed for recovery from the injection. Mice then performed the water maze tasks over a course of 8 weeks, at which point spatial acuity and contrast sensitivity were reassessed. A 
mouse performed a maximum of 5 sessions per day with activity modified based on signs of fatigue. At the beginning of each session mice were removed from their cage and placed in a holding cage lined with paper towels and illuminated with a $100-\mathrm{W}$ bulb that served as the heat source. Mice were returned to their holding cage after each trial and rested for 2-3 minutes before the next trial was performed.

Statistics. Data are presented as mean \pm SEM from at least 3 independent experiments. Sample size (retinas, eyes, mice) was determined on the basis of our previous studies $(12,22)$. The eyes showing signs of inflammation or cataract were excluded from analysis. Samples (retinas, eyes, or mice) were allocated to their experimental groups according to their genotype, and therefore there was no randomization. To compare the numbers between 2 experimental groups, we used a 2-tailed Student's $t$ test. Comparison between more than 2 groups was analyzed using 1-way ANOVA followed by Tukey's multiple comparisons test. $P$ values less than 0.05 were considered statistically significant.

Study approval. All animal procedures were in compliance with the NIH Guide for the Care and Use of Laboratory Animals (National Academies Press; 2011) and approved by the Institutional Animal Care and Use Committees at the State University of New York College of Optometry.

\section{Author contributions}

$\mathrm{AA}$ and $\mathrm{SAB}$ conceived and designed the study, performed data analysis, and wrote the manuscript. AA performed confocal microscopy and image analysis. SK performed induction of experimental glaucoma, conducted immunohistochemical experiments, and analyzed the data. SK, HR, and SV conducted and analyzed ERG and VEP experiments and wrote the corresponding section in the paper. KR performed behavioral experiments, performed data analysis, and genotyped the different mouse strains. All authors participated in the interpretation of results and provided critical reviews of the manuscript and final approval for submission.

\section{Acknowledgments}

This work was supported by NIH grants EYO07360 and EY026024 awarded to SAB.

Address correspondence to: Stewart A. Bloomfield, SUNY College of Optometry, 33 West 42nd Street, New York, New York 10036, USA. Phone: 212.938.5532; Email: sbloomfield@sunyopt.edu.
1. Quigley HA. Neuronal death in glaucoma. Prog Retin Eye Res. 1999;18(1):39-57.

2. Schwartz M. Neurodegeneration and neuroprotection in glaucoma: development of a therapeutic neuroprotective vaccine: the Friedenwald lecture. Invest Ophthalmol Vis Sci. 2003;44(4):1407-1411.

3. Chang EE, Goldberg JL. Glaucoma 2.0: neuroprotection, neuroregeneration, neuroenhancement. Ophthalmology. 2012;119(5):979-986.

4. Tian K, Shibata-Germanos S, Pahlitzsch M, Cordeiro MF. Current perspective of neuroprotection and glaucoma. Clin Ophthalmol. 2015;9:2109-2118.

5. Shih GC, Calkins DJ. Secondary neuroprotective effects of hypotensive drugs and potential mechanisms of action. Expert Rev Ophthalmol. 2012;7(2):161-175.

6. Kuehn MH, Fingert JH, Kwon YH. Retinal ganglion cell death in glaucoma: mechanisms and neuroprotective strategies. Ophthalmol Clin North Am. 2005;18(3):383-395.

7. Levkovitch-Verbin H, Quigley HA, KerriganBaumrind LA, D’Anna SA, Kerrigan D, Pease ME. Optic nerve transection in monkeys may result in secondary degeneration of retinal ganglion cells. Invest Ophthalmol Vis Sci. 2001;42(5):975-982.

8. Calkins DJ. Critical pathogenic events underlying progression of neurodegeneration in glaucoma. Prog Retin Eye Res. 2012;31(6):702-719.

9. Andrade-Rozental AF, Rozental R, Hopperstad MG, Wu JK, Vrionis FD, Spray DC. Gap junctions: the "kiss of death" and the "kiss of life.". Brain Res Brain Res Rev. 2000;32(1):308-315.

10. Decrock E, et al. Connexin-related signaling in cell death: to live or let die? Cell Death Differ. 2009;16(4):524-536.

11. Spray DC, Hanstein R, Lopez-Quintero SV, Stout RF, Suadicani SO, Thi MM. Gap junctions and bystander effects: Good Samaritans and executioners. Wiley Interdiscip Rev Membr Transp Signal. 2013;2(1):1-15.

12. Akopian A, et al. Gap junction-mediated death of retinal neurons is connexin and insult specific: a potential target for neuroprotection. J Neurosci. 2014;34(32):10582-10591.

13. Bloomfield SA, Völgyi B. The diverse functional roles and regulation of neuronal gap junctions in the retina. Nat Rev Neurosci. 2009;10(7):495-506.

14. Ripps H. Cell death in retinitis pigmentosa: gap junctions and the 'bystander' effect. Exp Eye Res. 2002;74(3):327-336.

15. Krysko DV, Leybaert L, Vandenabeele P, D'Herde K. Gap junctions and the propagation of cell survival and cell death signals. Apoptosis. 2005;10(3):459-469.

16. Das S, et al. Protection of retinal cells from ischemia by a novel gap junction inhibitor. Biochem Biophys Res Commun. 2008;373(4):504-508.

17. Kerr NM, Johnson CS, Zhang J, Eady EK, Green CR, Danesh-Meyer HV. High pressure-induced retinal ischaemia reperfusion causes upregulation of gap junction protein connexin 43 prior to retinal ganglion cell loss. Exp Neurol. 2012;234(1):144-152.

18. Lei Y, et al. Topography of neuron loss in the retinal ganglion cell layer in human glaucoma. $\mathrm{Br} \mathrm{J}$ Ophthalmol. 2009;93(12):1676-1679.

19. Reichstein D, Ren L, Filippopoulos T, Mittag T, Danias J. Apoptotic retinal ganglion cell death in the DBA/2 mouse model of glaucoma. Exp Eye Res. 2007;84(1):13-21.

20. May CA, Mittag T. Neuronal nitric oxide synthase (nNOS) positive retinal amacrine cells are altered in the DBA/2NNia mouse, a murine model for angle-closure glaucoma. J Glaucoma. 2004;13(6):496-499.

21. Moon JI, et al. Changes in retinal neuronal populations in the DBA/2J mouse. Cell Tissue Res. 2005;320(1):51-59.

22. Akopian A, Kumar S, Ramakrishnan H, Viswanathan S, Bloomfield SA. Amacrine cells coupled to ganglion cells via gap junctions are highly vulnerable in glaucomatous mouse retinas [published online ahead of print July 13, 2016]. J Comp
Neurol. https://doi.org/ 10.1002/cne.24074

23. Völgyi B, Chheda S, Bloomfield SA. Tracer coupling patterns of the ganglion cell subtypes in the mouse retina. JComp Neurol. 2009;512(5):664-687.

24. Sappington RM, Carlson BJ, Crish SD, Calkins DJ. The microbead occlusion model: a paradigm for induced ocular hypertension in rats and mice. Invest Ophthalmol Vis Sci. 2010;51(1):207-216.

25. Moinfar Z, Dambach H, Faustmann PM. Influence of drugs on gap junctions in glioma cell lines and primary astrocytes in vitro. Front Physiol. 2014;5:186

26. Pan F, Mills SL, Massey SC. Screening of gap junction antagonists on dye coupling in the rabbit retina. Vis Neurosci. 2007;24(4):609-618.

27. Inman DM, Horner PJ. Reactive nonproliferative gliosis predominates in a chronic mouse model of glaucoma. Glia. 2007;55(9):942-953.

28. Gallego BI, et al. IOP induces upregulation of GFAP and MHC-II and microglia reactivity in mice retina contralateral to experimental glaucoma. J Neuroinflammation. 2012;9:92.

29. Howell GR, et al. Axons of retinal ganglion cells are insulted in the optic nerve early in DBA/2J glaucoma. J Cell Biol. 2007;179(7):1523-1537.

30. Sanes JR, Masland RH. The types of retinal ganglion cells: current status and implications for neuronal classification. Annu Rev Neurosci. 2015;38:221-246.

31. Nadal-Nicolás FM, et al. Brn3a as a marker of retinal ganglion cells: qualitative and quantitative time course studies in naive and optic nerve-injured retinas. Invest Ophthalmol Vis Sci. 2009;50(8):3860-3868.

32. Jeon CJ, Strettoi E, Masland RH. The major cell populations of the mouse retina. JNeurosci. 1998;18(21):8936-8946.

33. Haverkamp S, Wässle H. Immunocytochemical analysis of the mouse retina. JComp Neurol. 2000;424(1):1-23.

34. Güldenagel M, et al. Expression patterns of connexin genes in mouse retina. JComp Neurol. 
2000;425(2):193-201.

35. Sun D, Lye-Barthel M, Masland RH, Jakobs TC. The morphology and spatial arrangement of astrocytes in the optic nerve head of the mouse. JComp Neurol. 2009;516(1):1-19.

36. Saszik SM, Robson JG, Frishman LJ. The scotopic threshold response of the dark-adapted electroretinogram of the mouse. J Physiol (Lond). 2002;543(pt 3):899-916.

37. Bui BV, Fortune B. Ganglion cell contributions to the rat full-field electroretinogram. J Physiol (Lond). 2004;555(pt 1):153-173.

38. Wachtmeister L. Oscillatory potentials in the retina: what do they reveal. Prog Retin Eye Res. 1998;17(4):485-521.

39. Holopigian K, Seiple W, Mayron C, Koty R, Lorenzo M. Electrophysiological and psychophysical flicker sensitivity in patients with primary open-angle glaucoma and ocular hypertension. Invest Ophthalmol Vis Sci. 1990;31(9):1863-1868.

40. Hancock HA, Kraft TW. Oscillatory potential analysis and ERGs of normal and diabetic rats. Invest Ophthalmol Vis Sci. 2004;45(3):1002-1008.

41. Prusky GT, Douglas RM. Characterization of mouse cortical spatial vision. Vision Res. 2004;44(28):3411-3418.

42. Prusky GT, West PW, Douglas RM. Behavioral assessment of visual acuity in mice and rats. Vision Res. 2000;40(16):2201-2209.

43. Murphy MC, et al. Retinal structures and visual cortex activity are impaired prior to clinical vision loss in glaucoma. Sci Rep. 2016;6:31464.

44. Bayer AU, et al. Electroretinographic abnormalities in a rat glaucoma model with chronic elevated intraocular pressure. Exp Eye Res. 2001;72(6):667-677.

45. Nickells RW. The cell and molecular biology of glaucoma: mechanisms of retinal ganglion cell death. Invest Ophthalmol Vis Sci.
2012;53(5):2476-2481.

46. El-Danaf RN, Huberman AD. Characteristic patterns of dendritic remodeling in early-stage glaucoma: evidence from genetically identified retinal ganglion cell types. JNeurosci. 2015;35(6):2329-2343.

47. Della Santina L, Inman DM, Lupien CB, Horner PJ, Wong RO. Differential progression of structural and functional alterations in distinct retinal ganglion cell types in a mouse model of glaucoma. J Neurosci. 2013;33(44):17444-17457.

48. Pang JJ, Frankfort BJ, Gross RL, Wu SM. Elevated intraocular pressure decreases response sensitivity of inner retinal neurons in experimental glaucoma mice. Proc Natl Acad Sci U S A. 2015;112(8):2593-2598.

49. Jakobs TC, Libby RT, Ben Y, John SW, Masland RH. Retinal ganglion cell degeneration is topological but not cell type specific in DBA/2J mice. JCell Biol. 2005;171(2):313-325.

50. Völgyi B, Abrams J, Paul DL, Bloomfield SA. Morphology and tracer coupling pattern of alpha ganglion cells in the mouse retina. JComp Neurol. 2005;492(1):66-77.

51. Hoge GJ, Davidson KG, Yasumura T, Castillo PE, Rash JE, Pereda AE. The extent and strength of electrical coupling between inferior olivary neurons is heterogeneous. J Neurophysiol. 2011;105(3):1089-1101.

52. Kothmann WW, Massey SC, O’Brien J. Dopamine-stimulated dephosphorylation of connexin 36 mediates AII amacrine cell uncoupling. J Neurosci. 2009;29(47):14903-14911.

53. Danesh-Meyer HV, Zhang J, Acosta ML, Rupenthal ID, Green CR. Connexin 43 in retinal injury and disease. Prog Retin Eye Res. 2016;51:41-68.

54. Danesh-Meyer HV, et al. Connexin 43 mimetic peptide reduces vascular leak and retinal ganglion cell death following retinal ischaemia. Brain. 2012;135(pt 2):506-520.

55. Kerr NM, Johnson CS, Green CR, Danesh-Meyer HV. Gap junction protein connexin 43 (GJA1) in the human glaucomatous optic nerve head and retina. J Clin Neurosci. 2011;18(1):102-108.

56. Deans MR, Volgyi B, Goodenough DA, Bloomfield SA, Paul DL. Connexin 36 is essential for transmission of rod-mediated visual signals in the mammalian retina. Neuron. 2002;36(4):703-712.

57. Völgyi B, Deans MR, Paul DL, Bloomfield SA. Convergence and segregation of the multiple rod pathways in mammalian retina. J Neurosci. 2004;24(49):11182-11192.

58. Güldenagel M, et al. Visual transmission deficits in mice with targeted disruption of the gap junction gene connexin36. J Neurosci. 2001;21(16):6036-6044.

59. Blankenship AG, et al. The role of neuronal connexins 36 and 45 in shaping spontaneous firing patterns in the developing retina. J Neurosci. 2011;31(27):9998-10008.

60. Pang JJ, Paul DL, Wu SM. Survey on amacrine cells coupling to retrograde-identified ganglion cells in the mouse retina. Invest Ophthalmol Vis Sci. 2013;54(8):5151-5162.

61. Chen H, et al. Optic neuropathy due to microbeadinduced elevated intraocular pressure in the mouse. Invest Ophthalmol Vis Sci. 2011;52(1):36-44.

62. Galindo-Romero C, et al. Axotomy-induced retinal ganglion cell death in adult mice: quantitative and topographic time course analyses. Exp Eye Res. 2011;92(5):377-387.

63. Ridder WH, Nusinowitz S. The visual evoked potential in the mouse - origins and response characteristics. Vision Res. 2006;46(6-7):902-913.

64. Gellermann LW. Chance orders of alternating stimuli in visual discrimination experiments. Pedagog Semin J Genet Psychol. 1933;42(1):206-208.

65. Brainard DH. The psychophysics toolbox. Spat Vis. 1997;10(4):433-436. 